INTERNATIONAL JOURNAL FOR NUMERICAL METHODS IN FLUIDS

Int. J. Numer. Meth. Fluids 2009; 59:147-171

Published online 2 June 2008 in Wiley InterScience (www.interscience.wiley.com). DOI: 10.1002/fld.1804

\title{
On surface tension modelling using the level set method
}

\author{
Sergey V. Shepel ${ }^{1, *, \dagger, \ddagger}$ and Brian L. Smith ${ }^{2}$ \\ ${ }^{1}$ EPFL Laboratory for Hydraulic Machines, Swiss Federal Institute of Technology, Ave. de Cour, 33 bis, CH-1007, \\ Lausanne, Switzerland \\ ${ }^{2}$ Thermal-Hydraulics Laboratory, Paul Scherrer Institute, CH-5232, Villigen PSI, Switzerland
}

\begin{abstract}
SUMMARY
The paper describes and compares the performance of two options for numerically representing the surface tension force in combination with the level set interface-tracking method. In both models, the surface tension is represented as a body force, concentrated near the interface, but the technical implementation is different: the first model is based on a traditional level set approach in which the force is distributed in a band around the interface using a regularized delta function, whereas in the second, the force is partly distributed in a band around the interface and partly localized to the actual computational cells containing the interface. A comparative study, involving analysis of several two-phase flows with moving interfaces, shows that in general the two surface tension models produce results of similar accuracy. However, in the particular case of merging and pinching-off of interfaces, the traditional level set model of surface tension produces an error that results in non-converging solutions for film-like interfaces (i.e. ones involving large contact areas). In contrast, the second model, based on the localized representation of the surface tension force, displays consistent first-order convergence. Copyright (C) 2008 John Wiley \& Sons, Ltd.
\end{abstract}

Received 25 September 2007; Revised 15 February 2008; Accepted 20 February 2008

KEY WORDS: level set; surface tension; convergence; film; finite element; finite volume

\section{INTRODUCTION}

The present work is concerned with the modelling of surface tension at inter-phase boundaries in viscous, isothermal, incompressible two-phase flows using the level set interface-tracking method. The phases are assumed to be immiscible. Stated briefly, the central idea of the level set method lies in its mathematical representation of the interface as the zero level set of a continuous scalar marker function $[1,2]$. The evolution of this marker function (traditionally called the level set function)

\footnotetext{
*Correspondence to: Sergey V. Shepel, Swiss Federal Laboratories for Materials Testing and Research (EMPA), CH-8600, Dübendorf, Switzerland.

†E-mail: sergey.shepel@empa.ch

$¥$ A part of the present study was carried out while the author was at Thermal-Hydraulics Laboratory, Paul Scherrer Institute, CH-5232, Villigen PSI, Switzerland.
} 
in space and time is governed by an advection equation, combined with a special renormalization procedure [1-3]. For the basics of the level set method, the interested reader is referred to the book by Sethian [2] and, for the latest state of research in this field, the recent review by Losasso et al. [4].

It should be noted that the level set method is one of several interface-tracking techniques used routinely for two-phase incompressible flow simulations. Others are the front-tracking method $[5,6]$, the boundary integral method [7], and the volume-of-fluid (VOF) method [8-10]. Unfortunately, it does not appear possible at the present time to assert which of the methods is generally superior, as each appears to have its own strengths and weaknesses. Discussion of the relative merits of the different approaches is beyond the scope of this study, but some information on this subject can be found, for instance, in a review of interface-tracking methods by Tryggvason et al. [11] and in the report by Kothe [12]. The main advantages of the level set method compared with other techniques include (1) the attractive simplicity of its mathematical formulation and programming, and (2) the ability of the method to simulate complex interfacial flows with strong surface tension effects.

In the level set method, the surface tension force, which is essentially a surface force, is traditionally modelled as a distributed body force, though concentrated in a band around the interfaces. Normally, the band is 2-4 computational cells wide and arranged in such a way that the force has a maximum on the interface and decays rapidly with distance from it $[1,2]$. Thus, the variation of the surface tension across the interface may be described in terms of a regularized (smoothed) delta function with compact support. This approach removes the interface singularity from the standard continuum fluid flow equations, and ultimately allows the surface tension to be modelled using standard numerical techniques on Eulerian grids. The jump in phase properties across the interface, such as density and viscosity, is correspondingly modelled using a regularized Heaviside function $[1,2]$.

An alternative approach is to model the jump in phase properties across the interface and the jump in pressure due to surface tension at the sub-cell scale. For instance, this can be done using the boundary condition capturing (BCC) technique of Liu et al. [13] (see also the recent work of Smereka [14]). Kang et al. [15] and Hong et al. [16] have constructed level set formulations based on this approach and demonstrated, by means of several benchmark simulations, that the use of the BCC method results in a lower intensity of parasitic currents (discussed below) and better resolution of interfacial instabilities compared with the regularized delta function method. On the other hand, the BCC technique is rather complicated in terms of programming, which makes it difficult to implement in general-purpose commercial computational fluid dynamics (CFD) software. For this reason, in this study the traditional level set approach involving regularization of the delta and Heaviside functions is employed.

The accuracy of the numerical integration associated with different regularized delta functions has been analysed by several groups [17-19]. In particular, Tornberg and Engquist [18] formulated several requirements that the regularized delta functions must satisfy in order to ensure that the error of integration is bounded by $O\left(h^{q}\right)$, where $h$ is the mesh size and $q$ a positive integer. One of these requirements states that the width of the support band for the delta function must be larger than $q h$. However, if the integrand function is resolved poorly, which happens, for example, when parts of the moving interface approach each other within a distance less than $q h$, the integration error can rise significantly. Such situations occur in applications involving the merging and pinching-off of interfaces, as well as in the development of film-like interfaces, since then the bands over which the surface tension is distributed overlap, leading to inaccurate calculation of the surface tension 
force. (Here, by film-like interfaces, we imply those inter-phase boundaries some parts of which are located within a short distance from each other over a large area. For instance, long thin ligaments that develop as a result of interfacial instabilities can be classified as film-like interfaces.)

Recently, Ilic et al. [20] reported results of a simulation of a bubble-train flow using the VOF method in which the surface tension was modelled as a body force, but localized only to those computational cells that actually contained the interface. The thickness of the band over which the surface force was distributed was then effectively reduced to just one cell. Apparently, the convergence rate of this surface tension model cannot be higher than first order, as the surface tension force is imposed not on the interface itself, but at the cell nodes, which are located at a distance $O(h)$ away from the interface. Nevertheless, the approach is of interest in the context of level set modelling, as it appears to avoid the lack of accuracy associated with overlapping regions of the distributed surface tension force. To our knowledge, a model analogous to that employed by Ilic et al. [20] has never been implemented before in level set formulations. In order to determine whether the use of the model leads to improved modelling capability compared with the standard model employing quadrature of the regularized delta function, a level set formulation that includes both surface tension models has been implemented into the commercial CFD code CFX-4 [21] and a series of benchmark simulations performed. A critical analysis of the results is given here.

\section{BASIC EQUATIONS AND NUMERICAL SCHEME}

\section{Finite-element/finite-volume level set formulation}

The local equations governing the motion of an unsteady, viscous, Newtonian, incompressible two-phase fluid are the Navier-Stokes equations, which in conservative dimensionless form are given as

$$
\begin{gathered}
\frac{\partial\left(\rho^{*} \mathbf{u}^{*}\right)}{\partial t^{*}}+\nabla \cdot\left(\rho^{*} \mathbf{u}^{*} \otimes \mathbf{u}^{*}\right)= \\
\frac{\partial \rho^{*}}{\partial t^{*}}+\nabla \cdot\left(\rho^{*} \mathbf{u}^{*}\right)=0
\end{gathered}
$$

The asterisk superscript indicates dimensionless variables, for which the following scaling applies:

$$
\begin{array}{cll}
\mathbf{u}^{*}=\mathbf{u} / u_{\mathrm{c}}, \quad \mathbf{x}^{*}=\mathbf{x} / l_{\mathrm{c}}, & t^{*}=t u_{\mathrm{c}} / l_{\mathrm{c}}, & \mathbf{g}^{*}=\mathbf{g} / g \\
\rho^{*}=\rho / \rho_{\mathrm{L}}, \quad \mu^{*}=\mu / \mu_{\mathrm{L}}, & p^{*}=p / \rho_{\mathrm{L}} u_{\mathrm{c}}^{2}, & \mathbf{B}^{*}=\mathbf{B} l_{\mathrm{c}}^{2} / \sigma
\end{array}
$$

Here, $\mathbf{u}$ is the velocity, $\mathbf{x}$ the distance, $t$ the time, $\mathbf{g}$ the acceleration of gravity, $\rho$ the density, $\mu$ the dynamic viscosity, $p$ the pressure, $\mathbf{B}$ the surface tension force, and $\sigma$ the surface tension coefficient. Additionally, $u_{\mathrm{c}}$ and $l_{\mathrm{c}}$ are characteristic velocity and distance scales, the subscripts $L$ and $G$ denote values corresponding to the liquid and gas phases, and the use of bold script signifies vector quantities. The dimensionless groups that appear in Equations (1) are the Reynolds, Froude, and Weber numbers, given by

$$
R e=\rho_{\mathrm{L}} u_{\mathrm{c}} l_{\mathrm{c}} / \mu_{\mathrm{L}}, \quad F r=u_{\mathrm{c}}^{2} / g l_{\mathrm{c}}, \quad W e=\rho_{\mathrm{L}} u_{\mathrm{c}}^{2} l_{\mathrm{c}} / \sigma
$$

The physical thickness of the liquid-gas interfacial region is of the order of several Angstrom [22] and in CFD applications is modelled simply as a surface discontinuity. However, in the level set 
method, the actual liquid-gas transition region is generally replaced by an artificial transition zone of thickness $2 \varepsilon$, where $\varepsilon=O(h)$ with $h$ being a measure of the mesh size, and this procedure is followed here. In the transition zone, the density and viscosity are assumed to vary monotonically from the values of the liquid phase to the values of the gas phase. Accordingly, the iso-surface $\left(\rho_{\mathrm{L}}+\rho_{\mathrm{G}}\right) / 2$ is defined as the geometric interfacial surface $\Gamma(\mathbf{x}, t)$. The surface tension force, $\mathbf{B}$, can also be distributed within the transition zone, analogously to the density and viscosity. The details of the modelling of $\mathbf{B}, \rho$, and $\mu$ at the interface are given in the following sections.

To simulate the motion of the interface, a special scalar function $\phi(\mathbf{x}, t)$ is introduced, the level set function, which is defined as follows: $\phi=-d(\mathbf{x}, t)$ if $\mathbf{x}$ lies in the liquid and $\phi=+d(\mathbf{x}, t)$ if $\mathbf{x}$ lies in the gas, where $d(\mathbf{x}, t)$ is the shortest distance from the point $\mathbf{x}$ to the interface $\Gamma$. Hence, the interface $\Gamma(\mathbf{x}, t)$ is associated with the zero level set of $\phi(\mathbf{x}, t)=0$. Formally, the position of the interface can be obtained from the solution of the advection equation:

$$
\frac{\partial \phi^{*}}{\partial t^{*}}+\mathbf{u}^{*} \cdot \nabla \phi^{*}=0
$$

where $\phi^{*}=\phi / l_{\mathrm{c}}$ is the dimensionless equivalent of the level set function. Considering the density of the phase mixture to be a function of $\phi$ according to $\rho(\mathbf{x}, t)=\rho(\phi(\mathbf{x}, t))$, by virtue of Equation (4), the mass continuity condition equation (1b) reduces to that of an incompressible liquid: $\nabla \cdot \mathbf{u}^{*}=0$.

To maintain the level set function, $\phi(\mathbf{x}, t)$, as the signed distance function $d(\mathbf{x}, t)$, it needs to be reinitialized at every time step [1,2]. An efficient reinitialization procedure has been proposed by Sussman and Fatemi [3] based on finding the steady-state solution of the following equation:

$$
\frac{\partial \phi^{*}}{\partial \tau^{*}}+\operatorname{sign}\left(\phi_{0}^{*}\right)\left(\left|\nabla \phi^{*}\right|-1\right)=\lambda \delta_{\varepsilon}^{*}\left(\phi^{*}\right)\left|\nabla \phi^{*}\right|
$$

where $\tau^{*}$ is a time-like variable (used only to approach the steady-state solution), $\phi_{0}$ is the initial distribution of the level set function, $\delta_{\varepsilon}^{*}\left(\phi^{*}\right)$ is a regularized delta function, and $\lambda$ is a correction coefficient calculated in such a way as to ensure mass conservation up to the first-order term in the Taylor expansion of the integral $\partial_{\tau} \int_{\Omega} H_{\varepsilon}\left(\phi^{*}\right) \mathrm{d} \Omega^{*}$ (see [3]). Here, $H_{\varepsilon}\left(\phi^{*}\right)$ is the regularized Heaviside function, defined as

$$
H_{\varepsilon}(\gamma)=\int_{-\infty}^{\gamma} \delta_{\varepsilon}(\chi) \mathrm{d} \chi
$$

The numerical algorithm is constructed as follows: Equations (1) are solved using the known level set distribution, $\phi\left(\mathbf{x}, t_{n}\right)$, to obtain the velocity field at the next time level $\mathbf{u}\left(\mathbf{x}, t_{n+1}\right)$. This in turn is used to update the level set distribution via Equation (4). The obtained solution, $\phi_{0}\left(\mathbf{x}, t_{n+1}\right)$, will no longer be the signed distance to the interface; therefore, it is reinitialized by solving Equation (5) to steady state. This gives the normalized level set field, $\phi\left(\mathbf{x}, t_{n+1}\right)$.

As we are generally interested in modelling interfacial flows in complex geometries, and in both two and three dimensions, we choose the streamline-upwind/Petrov-Galerkin (SUPG) finiteelement (FE) method for solving the level set equations (4) and (5). This method is second-order accurate, robust, and readily adapted to three-dimensional, non-regular grids [23].

In the CFX-4 finite-volume (FV) solver of the Navier-Stokes equations (1), all variables, including velocity, pressure, and bulk body forces, are defined at cell centres. Boundary conditions are imposed at the so-called boundary nodes, which are located at the centres of the cell faces bordering the fluid domain. The code CFX-4 uses structured, body-fitted grids with hexahedral 


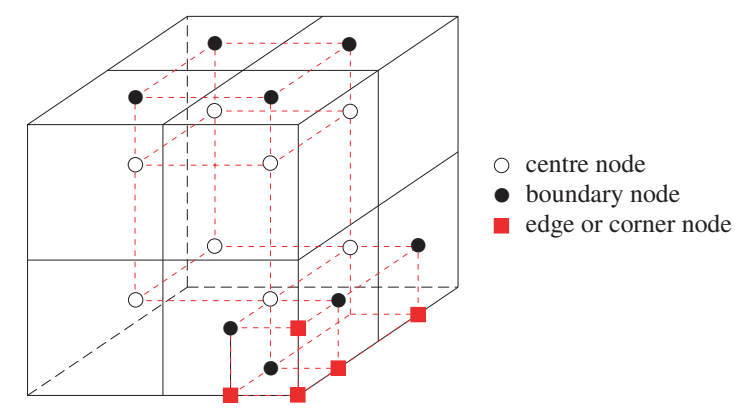

Figure 1. Schematic of grids, nodes, and elements for the construction of the FE mesh by overlaying the FV grid. The dashed lines show the edges of the FEs. Note that the construction of the FE mesh requires the introduction of new types of nodes not present in the FV mesh. The new nodes are placed on the edges and corners of the external boundaries of the fluid domain. Velocities at such nodes are prescribed, based on the boundary conditions.

computational cells. However, in the SUPG FE method, all variables are defined at element vertices. In order to avoid the necessity of interpolation between cell vertices and centres, which would inevitably result in smearing of the solution, we introduce a separate FE mesh, whose vertices are constructed from the centre and boundary nodes of the FV mesh. Thus, the fluid flow equations (1) are solved on the FV mesh, whereas the FE mesh is used to solve the level set equations (4) and (5). This idea is illustrated in Figure 1. The FEs are hexahedral and isoparametric with bilinear shape functions. The discretized equations (1) are solved using a second-order accurate nested (inner and outer) iteration technique based on the SIMPLEC velocity-pressure coupling algorithm [24].

It is important to point out that in CFX-4, the Navier-Stokes equations (1) are solved in conservative form, whereas the SUPG FE method is non-conservative. Hence, the resulting numerical scheme is a mixed FE-FV formulation. It was implemented into CFX-4 via the standard set of user subroutines. For a more detailed description of the numerical scheme and results from other validation studies, the reader is referred to our earlier papers $[25,26]$.

\section{Modelling at the interface}

Surface tension. The surface tension force on the interface is given by $\left.\mathbf{B}\right|_{\Gamma}=\sigma \kappa \mathbf{n}$, where $\mathbf{n}$ and $\kappa$ are, respectively, the interface normal vector and the curvature. In the level set method, these quantities are calculated as $\mathbf{n}=\nabla \phi /|\nabla \phi|$ and $\kappa=\nabla \cdot(\nabla \phi /|\nabla \phi|)$, which offers an elegant way of generalization of the localized surface tension force acting on the interfacial surface $\Gamma(\mathbf{x}, t)$ as a distributed body force. This approach has been employed routinely in the traditional level set formulations by distributing the surface tension force in a band around the interface

$$
\mathbf{B}=\sigma \kappa(\phi) \mathbf{n}(\phi) \delta_{\varepsilon}(\phi)
$$

with the maximum occurring on the interface $[1,2]$. The use of $\delta_{\varepsilon}(\phi)$ in Equation (7) is actually a generalization of the one-dimensional regularized delta function to higher dimensions. The width of the support region of $\delta_{\varepsilon}(\phi)$ is characterized by $\varepsilon$, the half-thickness of the band. Typically, the value of $\varepsilon$ is set to $h$ or $2 h[1,3]$. Hereafter, we refer to this surface tension model as Method A. Details of the delta functions $\delta_{\varepsilon}(\phi)$ employed in this study are given in Appendix A. 
In the model employed by Ilic et al. [20] for constructing a VOF interface-tracking method, the surface tension force is defined at the centres of those computational cells that are actually crossed by the interface. This approach can be adapted to the level set method as follows:

$$
\mathbf{B}=\sigma \kappa(\phi) \mathbf{n}(\phi) \Gamma_{\text {in }}
$$

where $\Gamma_{\text {in }}$ is the volumetric interfacial area concentration defined as $\Gamma_{\text {in }}=\Delta a / \Delta V$, with $\Delta a$ the area of the interface segment confined within a cell of volume $\Delta V$. The value of $\Delta a$ is calculated by approximating the interface within each cell as a plane with normal $\mathbf{n}$, located at distance $|\phi|$ from the cell centre. In our study, we incorporate this model into the FE-FV level set method and refer to it hereafter as Method B.

During preliminary tests involving CFD applications with the surface tension being the main driving force for the fluid motion, we found that the use of Method B, as represented in Equation (8), can lead to slow convergence and occasionally to inaccurate solutions. The principal difficulty appeared to arise from the abrupt change in the surface tension distribution as the interface moves from one computational cell to another. To illustrate the difficulty, consider the two FV cells shown in Figure 2(a). In cell $i$, the interface is located close to the right vertical cell edge and is almost parallel to it. If this interface, moving to the right, crosses the cell edge in one time step, the surface tension force at nodes $i$ and $j$, as given by Equation (8), changes in a discontinuous manner. Ultimately, this situation results in the above-mentioned poor convergence and lack of accuracy.

Consequently, in order to make Method B capable of handling flows with strong surface tension effects, it is necessary to adjust it with some means of smoothing the surface tension distribution, $\mathbf{B}$, around the interface. We have developed a straightforward smoothing procedure that can be applied easily on rectangular grids (this procedure can be also applied without modifications on unstructured grids; however, this issue is beyond the scope of this study). Consider the segment of the interface located between the centre nodes of the two FV cells $i$ and $j$, for which $\phi_{i} \leqslant 0$ and $\phi_{j}>0$ (see Figure 2). This interface segment can be located entirely in cell $i$ (as shown in Figure 2(a)), entirely in cell $j$ (Figure 2(b)), or in both cells $i$ and $j$, (as shown in Figure 2(c)). Denote the surface tension contributions at nodes $i$ and $j$ given by Equation (8) as $\mathbf{B}_{i}$ and $\mathbf{B}_{j}$, respectively. In order to make the surface tension distribution less sensitive to the movement of the interface across such cell edges, the contributions $\mathbf{B}_{i}$ and $\mathbf{B}_{j}$ are replaced with new values, denoted here as $\tilde{\mathbf{B}}_{i}$ and $\tilde{\mathbf{B}}_{j}$, which are calculated based on the proximity of the interface to the nodes. This procedure is applied only if the angle between the normal to the interface, $\mathbf{n}$, and the normal to the edge, $\mathbf{n}_{\mathrm{ED}}$, is less than a specified value $\alpha_{\mathrm{S}}$ :

$$
\begin{aligned}
\tilde{\mathbf{B}}_{i} & =(1-\xi)\left(\mathbf{B}_{i}+\mathbf{B}_{j}\right) \\
\tilde{\mathbf{B}}_{j} & =\xi\left(\mathbf{B}_{i}+\mathbf{B}_{j}\right)
\end{aligned} \quad \text { if } \cos ^{-1}\left(\mathbf{n} \cdot \mathbf{n}_{\mathrm{ED}}\right) \leqslant \alpha_{\mathrm{S}}
$$

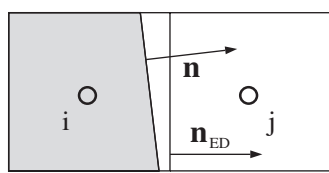

(a)

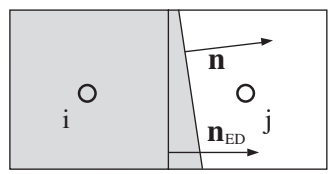

(b)

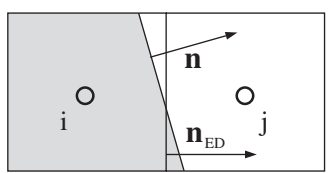

(c)

Figure 2. Three possible orientations of the interface in FV cells. The region occupied by the liquid phase is shown in grey: $\phi_{i} \leqslant 0, \phi_{j}>0$. 
where

$$
\xi=\frac{V_{i}\left(F_{i}-1 / 2\right)+V_{j} F_{j}}{V_{i} / 2+V_{j} / 2}, \quad \phi_{i} \leqslant 0, \quad \phi_{j}>0
$$

Here, $V_{i}$ and $V_{j}$ are the respective cell volumes, and $F_{i}$ and $F_{j}$ are the volume fractions of the liquid phase in cells $i$ and $j$ obtained by integration over the cell volume: $F_{i}=\left(1 / V_{i}\right) \int_{V_{i}} H(-\phi) \mathrm{d} \Omega$, where $H(\gamma)$ is the discontinuous Heaviside function. Later, we refer to Method B, supplemented with the smoothing procedure defined in Equations (9) and (10), as Modified Method B.

Clearly, increasing the value of $\alpha_{S}$ increases the number of cells over which the surface tension force is distributed. In the limit, by setting $\alpha_{S}=45^{\circ}$, one redistributes the surface tension force according to Equations (9) and (10) in all cells containing the interface, so that $\mathbf{B}$ is distributed in a band two cells wide. Note, however, that in this case the surface tension distribution generated by Modified Method B will not be identical to that generated by Method A with $\varepsilon=h$, which also distributes the surface tension in a band two cells wide. In fact, there can be a significant difference in accuracy of representation of the surface tension using the two models. This issue is addressed in the discussion of the results.

Density and viscosity. To aid numerical stability, the density and viscosity of the two-phase fluid are interpolated across the interface using the same regularized Heaviside function as follows:

$$
\begin{aligned}
& \rho^{*}\left(\mathbf{x}^{*}, t^{*}\right)=1+\left(\rho_{\mathrm{G}} / \rho_{\mathrm{L}}-1\right) H_{\varepsilon}\left(\phi^{*}\left(\mathbf{x}^{*}, t^{*}\right)\right) \\
& \mu^{*}\left(\mathbf{x}^{*}, t^{*}\right)=1+\left(\mu_{\mathrm{G}} / \mu_{\mathrm{L}}-1\right) H_{\varepsilon}\left(\phi^{*}\left(\mathbf{x}^{*}, t^{*}\right)\right)
\end{aligned}
$$

Equation (11a) follows directly from the assumption that the volume fractions of the gas and liquid phases in the neighbourhood of the interface are given, respectively, by $H_{\varepsilon}\left(\phi^{*}\left(\mathbf{x}^{*}, t^{*}\right)\right)$ and $\left(1-H_{\varepsilon}\left(\phi^{*}\left(\mathbf{x}^{*}, t^{*}\right)\right)\right)$. However, in general, the mixture viscosity is not given only in terms of the phase fractions. For this reason, Equation (11b) should be viewed as one possible way of local averaging, which, although popular with the level set and VOF communities, is by no means unique [12,27]. In particular, as pointed out by Kothe [12], the averaging of viscosities using Equation (11b) can result in a relatively high level of parasitic currents in the case in which $\mu_{\mathrm{L}}>>\mu_{\mathrm{G}}$. (Parasitic currents are discussed below.)

According to Equation (6), the value of $H_{\varepsilon}(\phi)$ grows from 0 to 1 in the band $-\varepsilon \leqslant \phi \leqslant \varepsilon$, where $\varepsilon=O(h)$. The delta function $\delta_{\varepsilon}(\phi)$ is normally chosen to be symmetric with respect to its argument, which gives $H_{\varepsilon}(0)=\frac{1}{2}$. Thus, in the limit of approaching the interface at $\phi(\mathbf{x})=0$ from the gas or liquid side, the local error in density and correspondingly pressure is $O(1)$ in the $L_{\infty}$ norm. However, it is reasonable to assume that in $N$-dimensional problems the number of cells crossed by the interface is $O\left(h^{-(N-1)}\right)$, whereas the number of all cells in the domain is $O\left(h^{-N}\right)$. Consequently, the overall error of the level set solution must be close to $O(h)$ in the $L_{2}$ norm. Indeed, as discussed in our earlier papers $[25,26]$, despite the fact that the FE-FV level set method was equipped with second-order solvers for all the governing equations (1), (4), and (5), the convergence rate of the solution in CFD applications, as measured in the $L_{2}$ norm, oscillated in the range $O(h)$ and $O\left(h^{2}\right)$, so that full second-order convergence was never achieved. Consequently, in CFD applications, the lack of accuracy associated with interpolation of density using the regularized Heaviside function given by Equation (11a) can offset the benefits obtained by using more accurate surface tension models if they are of higher accuracy than first order. This needs to be taken into account when analysing the results of the CFD simulations. 


\section{RESULTS AND DISCUSSION}

In this section, we apply the FE-FV level set formulation incorporating the two surface tension models to several selected two-dimensional and axisymmetric problems, with the purpose of testing and validating the two approaches vis à vis. In particular, Modified Method B is tested for different values of the model parameter $\alpha_{S}$.

The half-thickness of the compact support band for density and viscosity, as defined in Equations (11), is generally set to $\varepsilon=2 h$, as this value provides robust convergence. Here, the half-thickness of the surface tension support band, $\varepsilon_{\sigma}$, used in Equation (7), is varied between $h$ and $2 h$ to study its effect on the accuracy of the solution. The Navier-Stokes equations (1) are solved using a second-order scheme in both space and time. All simulations here have been carried out using uniform grids; see [26] for examples of applications of our FE-FV level set formulation using non-uniform grids.

The level set method is often challenged on the issue of mass conservation. Indeed, solving the level set equations (4) and (5) does not guarantee conservation of the fluid mass, as formally conservation of $\phi$ does not imply conservation of mass. Typically, in transient level set simulations, the fluid mass varies with time; however, these variations can be significantly minimized by using special numerical procedures [3, 28, 29].

\section{Film-like interface}

In this section, the two models are employed to calculate the surface tension force, $\mathbf{B}$, on the closed two-dimensional contour shown in Figure 3(a), the shape of which is reminiscent of a gas bubble rising in a fluid (e.g. $[1,30])$. The chosen contour consists of four $180^{\circ}$ arcs: two large arcs of radii $R_{o}$ and $R_{i}$ and two small arcs of radius $\left(R_{o}-R_{i}\right) / 2$ each. The coefficient of surface tension is set to $\sigma=1$. As an illustration, Figure 3(b) shows a numerical reconstruction of this contour for $R_{o}-R_{i}=0.125$ at the poor resolution of $h=0.1$. The values of the level set function, $\phi$, are specified at cell centres, as is done in the FV method, whereas the interface is, according to Method B, approximated by plane segments within each cell.

In order to estimate the separate contributions of the large and small arcs to the overall surface tension force, the computational domain is divided into two parts by a horizontal line passing

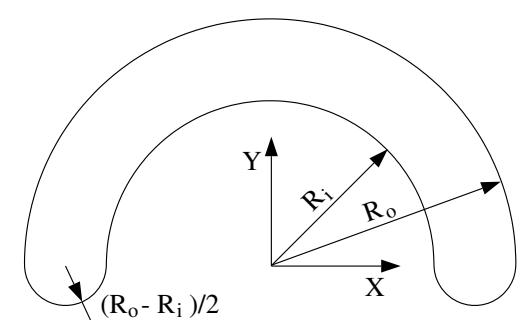

(a)

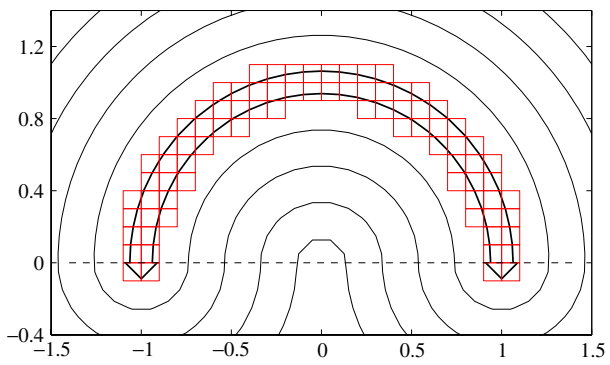

(b)

Figure 3. Calculation of the surface tension force on a closed contour: (a) schematic of the contour, and (b) reconstruction of the contour for $\left(R_{0}-R_{i}\right)=0.125$ and $h=0.1$. The contour shown with the bold line is approximated by plane segments within each cell. The FV cells crossed by the contour are indicated explicitely. The black continuous lines are the iso-lines of $\phi$, spaced $\Delta x=0.2$ apart. 
through the origin of the coordinate system and those points where the arcs are connected to each other (the dashed line in Figure 3(b)). As the contour is symmetric with respect to the $y$-axis, the horizontal component of $\mathbf{B}$ is not considered in the analysis, whereas the vertical component is calculated as follows: $B_{y}=h^{2} \sum_{i, j} \mathbf{J} \cdot \mathbf{B}\left(\mathbf{x}_{i, j}\right)$, where $\mathbf{J}=(0,1)$ is the unit vector along the $y$-axis. We denote the value of $B_{y}$ calculated in the upper part of the domain, i.e. for $y \geqslant 0$, as $B_{y}^{+}$and that in the lower part of the domain $(y \leqslant 0)$ as $B_{y}^{-}$. Note that Method B and Modified Method B provide identical results for the value of the net surface tension force, so that in this particular application we do not differentiate between the two methods when discussing the results.

The values of the arc radii are set to $R_{o}=1+\Delta R / 2$ and $R_{i}=1-\Delta R / 2$, so that the difference between the two is equal to $\Delta R$. The exact solution to this problem obtained by contour integration is given by $B_{y}^{+}=-4$ and $B_{y}^{-}=+4$. Theoretically, the net surface tension force on the closed contour is 0 , though the values of $B_{y}^{+}$and $B_{y}^{-}$obtained numerically do not exactly cancel each other out due to discretization errors. The integration errors for the convergence study may be defined as follows: $E_{B}^{+}=\left(B_{y}^{+}+4\right) / 4$ and $E_{B}^{-}=\left(B_{y}^{-}-4\right) / 4$. Then, the net force on the contour is given by $4\left(E_{B}^{+}+E_{B}^{-}\right)$.

The surface tension force $\mathbf{B}$ in Method $\mathrm{A}$ is modelled using the three different regularized delta functions $\delta_{\varepsilon_{\sigma}}(\phi)$ given in Appendix A. These are the most popular functions in the context of the level set modelling $[2,18]$. In order to evaluate the effect of thickness of the surface tension band on the solution of Method A, two values of $\varepsilon_{\sigma}$ are used: $h$ and $2 h$. Note that $\varepsilon_{\sigma}=h$ ensures the sharpest possible resolution of the surface tension force. In both Methods A and B, the first- and second-order derivatives of $\phi(\mathbf{x})$, which are necessary for calculating the interfacial gradient $\mathbf{n}$ and curvature $\kappa$, are computed numerically using second-order accurate central or one-sided stencils, as appropriate. The choice between the stencils is made using the procedure of minimization of divided differences [31].

Table I shows the results of the convergence study for $\Delta R=0.125$ for successively refined grids. Consistent convergence for Method A is achieved, provided the product of the interfacial curvature $\left(\kappa=16\right.$ for $\left.B_{y}^{-}\right)$and the mesh size $h$ satisfies the condition: $h \kappa \leqslant 0.2$ for $\varepsilon_{\sigma}=h$ and $h \kappa \leqslant 0.4$ for $\varepsilon_{\sigma}=2 h$, which is actually a grid resolution criterion. An additional condition for ensuring consistent convergence of Method $\mathrm{A}$ is the requirement that the width of the compact support region of $\delta_{\varepsilon_{\sigma}}(\phi)$ is thin enough: specifically, $\left(R_{o}-R_{i}\right) / 2 \varepsilon_{\sigma} \geqslant 1$. If these two conditions are satisfied, Method A displays second-order convergence if used together with the cosine and linear hat delta functions, and close to second-order convergence for the $\delta_{\varepsilon\left(\nabla \phi, \varepsilon_{\sigma}\right)}^{\mathrm{L}}(\phi)$ function defined in Equation (A4). In contrast, Method B displays consistent first-order convergence starting from the coarsest mesh with $h=0.1$.

To see more clearly the effect of grid refinement on the performance of Methods A and B for poorly resolved interfaces with $\left(R_{o}-R_{i}\right) / 2 \varepsilon_{\sigma}<1$, we perform an additional convergence study for the same contour shown in Figure 3(a), but with the distance between the interfaces, $\Delta R$, scaled to the mesh size, $h$, as follows: $\Delta R=3 h / 2$. The results are given in Table II. As one can see, in this particular case, Method B displays consistent first-order convergence, whereas Method A does not converge at all, regardless of the chosen delta function $\delta_{\varepsilon_{\sigma}}(\phi)$.

We have obtained an analytical estimate for the integration error (due to overlapping surface tension bands) in the value of $\mathbf{B}$ produced by Method A with $\varepsilon_{\sigma}=h$ for film-like interfaces. The derivation is given in Appendix B. Using Equation (B2), one can easily estimate the error $E_{B}^{+}$ for the present application as $\sigma(2-\Delta R / h)^{2} / 8$, where $h \leqslant \Delta R \leqslant 2 h$. This estimate was obtained assuming that the surface tension force, $\mathbf{B}$, is calculated from Equation (7) using the linear hat 
Table I. Integration errors for the contour shown in Figure 3(a) with $\left(R_{o}-R_{i}\right)=0.125$.

\begin{tabular}{|c|c|c|c|c|c|c|c|}
\hline & $\varepsilon_{\sigma}$ & Error & 0.10 & $5.00 \times 10^{-2}$ & $\begin{array}{c}h \\
2.50 \times 10^{-2}\end{array}$ & $1.25 \times 10^{-2}$ & $6.25 \times 10^{-3}$ \\
\hline \multirow[t]{2}{*}{ Method A, $\delta_{\varepsilon\left(\nabla \phi, \varepsilon_{\sigma}\right)}^{\mathrm{L}}$} & $h$ & $\begin{array}{l}E_{B}^{+} \\
E_{B}^{-}\end{array}$ & $\begin{array}{c}1.55 \times 10^{-1} \\
-5.86 \times 10^{-1}\end{array}$ & $\begin{array}{c}1.08 \times 10^{-2} \\
-3.18 \times 10^{-1}\end{array}$ & $\begin{array}{c}1.52 \times 10^{-3} \\
-3.99 \times 10^{-3}\end{array}$ & $\begin{array}{c}-1.54 \times 10^{-4} \\
1.48 \times 10^{-2}\end{array}$ & $\begin{array}{c}-5.05 \times 10^{-5} \\
4.86 \times 10^{-3}\end{array}$ \\
\hline & $2 h$ & $\begin{array}{l}E_{B}^{+} \\
E_{B}^{-}\end{array}$ & $\begin{array}{c}3.03 \times 10^{-1} \\
-5.71 \times 10^{-1}\end{array}$ & $\begin{array}{c}1.39 \times 10^{-1} \\
-3.87 \times 10^{-1}\end{array}$ & $\begin{array}{c}4.07 \times 10^{-3} \\
-7.18 \times 10^{-2}\end{array}$ & $\begin{array}{c}-1.38 \times 10^{-4} \\
1.78 \times 10^{-2}\end{array}$ & $\begin{array}{c}-4.96 \times 10^{-5} \\
4.72 \times 10^{-3}\end{array}$ \\
\hline \multirow[t]{2}{*}{ Method A, $\delta_{\varepsilon_{\sigma}}^{\mathrm{L}}$} & $h$ & $\begin{array}{l}E_{B}^{+} \\
E_{B}^{-}\end{array}$ & $\begin{array}{c}9.69 \times 10^{-2} \\
-5.91 \times 10^{-1}\end{array}$ & $\begin{array}{c}8.55 \times 10^{-3} \\
-2.72 \times 10^{-1}\end{array}$ & $\begin{array}{c}8.26 \times 10^{-4} \\
-1.56 \times 10^{-2}\end{array}$ & $\begin{array}{c}-1.71 \times 10^{-4} \\
1.69 \times 10^{-2}\end{array}$ & $\begin{array}{c}-4.61 \times 10^{-5} \\
4.94 \times 10^{-3}\end{array}$ \\
\hline & $2 h$ & $\begin{array}{l}E_{B}^{+} \\
E_{B}^{-}\end{array}$ & $\begin{array}{c}2.58 \times 10^{-1} \\
-5.77 \times 10^{-1}\end{array}$ & $\begin{array}{c}7.94 \times 10^{-2} \\
-3.44 \times 10^{-1}\end{array}$ & $\begin{array}{c}1.32 \times 10^{-3} \\
-4.12 \times 10^{-2}\end{array}$ & $\begin{array}{c}-8.69 \times 10^{-5} \\
1.73 \times 10^{-2}\end{array}$ & $\begin{array}{c}-2.01 \times 10^{-5} \\
4.72 \times 10^{-3}\end{array}$ \\
\hline \multirow[t]{2}{*}{ Method A, $\delta_{\varepsilon_{\sigma}}^{\mathrm{cos}}$} & $h$ & $\begin{array}{l}E_{B}^{+} \\
E_{B}^{-}\end{array}$ & $\begin{array}{c}7.32 \times 10^{-2} \\
-5.96 \times 10^{-1}\end{array}$ & $\begin{array}{c}8.21 \times 10^{-3} \\
-2.50 \times 10^{-1}\end{array}$ & $\begin{array}{c}1.02 \times 10^{-3} \\
-1.20 \times 10^{-2}\end{array}$ & $\begin{array}{c}-8.73 \times 10^{-5} \\
1.88 \times 10^{-2}\end{array}$ & $\begin{array}{c}-2.53 \times 10^{-5} \\
5.25 \times 10^{-3}\end{array}$ \\
\hline & $2 h$ & $\begin{array}{l}E_{B}^{+} \\
E_{B}^{-}\end{array}$ & $\begin{array}{c}2.37 \times 10^{-1} \\
-5.81 \times 10^{-1}\end{array}$ & $\begin{array}{c}4.88 \times 10^{-2} \\
-3.28 \times 10^{-1}\end{array}$ & $\begin{array}{c}1.22 \times 10^{-3} \\
-3.26 \times 10^{-2}\end{array}$ & $\begin{array}{c}-8.75 \times 10^{-5} \\
1.70 \times 10^{-2}\end{array}$ & $\begin{array}{c}-2.27 \times 10^{-5} \\
4.74 \times 10^{-3}\end{array}$ \\
\hline Method B & N/A & $\begin{array}{l}E_{B}^{+} \\
E_{B}^{-}\end{array}$ & $\begin{array}{c}4.89 \times 10^{-2} \\
-5.48 \times 10^{-1}\end{array}$ & $\begin{array}{c}8.37 \times 10^{-3} \\
-2.31 \times 10^{-1}\end{array}$ & $\begin{array}{c}1.26 \times 10^{-3} \\
-9.18 \times 10^{-3}\end{array}$ & $\begin{array}{c}-4.15 \times 10^{-4} \\
2.56 \times 10^{-2}\end{array}$ & $\begin{array}{c}-1.45 \times 10^{-4} \\
9.62 \times 10^{-3}\end{array}$ \\
\hline
\end{tabular}

The errors have been computed by averaging the predictions of $B_{y}$ over 64 small shifts in the position of the grid with respect to the contour.

Table II. Integration errors $E_{B}^{+}$for the contour shown in Figure 3(a), with the thickness of the contour scaled to the mesh size according to $\Delta R=3 h / 2$. Here, $\varepsilon_{\sigma}=h$.

\begin{tabular}{lccccc}
\hline & 0.10 & $5.00 \times 10^{-2}$ & $2.50 \times 10^{-2}$ & $1.25 \times 10^{-2}$ & $6.25 \times 10^{-3}$ \\
\hline Method A, $\delta_{\varepsilon(\nabla \phi, h)}^{\mathrm{L}}$ & $1.11 \times 10^{-1}$ & $9.85 \times 10^{-2}$ & $9.15 \times 10^{-2}$ & $8.82 \times 10^{-2}$ & $8.68 \times 10^{-2}$ \\
Method A, $\delta_{h}^{\mathrm{L}}$ & $5.67 \times 10^{-2}$ & $4.43 \times 10^{-2}$ & $3.69 \times 10^{-2}$ & $3.34 \times 10^{-2}$ & $3.19 \times 10^{-2}$ \\
Method A, $\delta_{h}^{\cos }$ & $4.05 \times 10^{-2}$ & $2.71 \times 10^{-2}$ & $1.91 \times 10^{-2}$ & $1.55 \times 10^{-2}$ & $1.37 \times 10^{-2}$ \\
Method B & $2.95 \times 10^{-2}$ & $1.59 \times 10^{-2}$ & $7.07 \times 10^{-3}$ & $3.32 \times 10^{-3}$ & $1.47 \times 10^{-3}$ \\
\hline
\end{tabular}

The errors have been computed by averaging the predictions of $B_{y}$ over 64 small shifts in the position of the grid with respect to the contour.

delta function $\delta_{\varepsilon_{\sigma}}^{\mathrm{L}}(\phi)$ defined in Equation (A3); however, the use of other delta functions should lead to similar error estimates. We note that the value of the error depends on the ratio $\Delta R / h$ as well as the surface tension coefficient, $\sigma$. However, the error does not approach 0 as the grid is refined; this agrees with the results obtained numerically. 
Later in this study, the surface tension force in Method A is approximated via Equation (7) using the cosine regularized delta function $\delta_{\varepsilon_{\sigma}}^{\cos }(\phi)$ defined in Equation (A2). This function was chosen for its superior accuracy, as observed in this study and in our earlier investigations [25, 26]. Accordingly, the function $\delta_{\varepsilon}^{\cos }(\phi)$ is employed in Equation (6) for the construction of the regularized Heaviside function, $H_{\varepsilon}(\phi)$, for approximating the jumps in density and viscosity at the interface.

\section{Liquid drop in equilibrium}

Here, we present a simulation of a drop of water with radius $R=2 \mathrm{~cm}$ in stagnant air in the absence of gravity. Under these conditions, the drop should acquire a spherical shape and remain in equilibrium with a jump in pressure across the surface given by Laplace's formula $\Delta p=2 \sigma / R$. The properties of water are set to $\rho_{\mathrm{L}}=10^{3} \mathrm{~kg} / \mathrm{m}^{3}, \mu_{\mathrm{L}}=10^{-3} \mathrm{~kg} / \mathrm{m} \mathrm{s}$, with the water/air density and viscosity ratios equal to $\rho_{\mathrm{L}} / \rho_{\mathrm{G}}=860$ and $\mu_{\mathrm{L}} / \mu_{\mathrm{G}}=54$; the surface tension is $\sigma=0.072 \mathrm{~N} / \mathrm{m}$. An analogous problem, but for a planar two-dimensional drop, was considered by Francois et al. [32] using a second-order VOF method based on the piecewise linear interface calculation (PLIC) reconstruction procedure.

As a consequence of axial symmetry, the problem is modelled in cylindrical coordinates; the computational domain $\Omega_{\mathrm{D}}$ representing only half of the drop. The size of $\Omega_{\mathrm{D}}$ is chosen to be $6 \mathrm{~cm} \times 3 \mathrm{~cm}$. The increase in size of the chosen domain has been found to have a negligibly small effect on the solution. The air pressure at the domain external boundary is set to 0 . The simulation is run for one time step $\Delta t=10^{-6} \mathrm{~s}$. According to Laplace's formula, the exact solution to this problem is the discontinuous pressure distribution $p_{\text {exact }}(\mathbf{x})$ equal to 0 outside the drop and $7.2 \mathrm{~Pa}$ inside the drop, with a jump at the drop surface. Denoting the pressure inside the drop as $p_{\mathrm{IN}}$, we characterize the accuracy of the numerical solution according to the error estimates introduced by Brackbill et al. [8]:

$$
\begin{gathered}
E_{2}^{P}=\left(\int_{\Omega_{\mathrm{D}}}\left(p-p_{\text {exact }}\right)^{2} /\left(A_{\mathrm{D}} p_{\mathrm{IN}}^{2}\right) \mathrm{d} \Omega\right)^{1 / 2} \\
E_{\Delta P}=\int_{\Omega_{\mathrm{IN}}}\left(p-p_{\mathrm{IN}}\right) /\left(A_{\mathrm{IN}} p_{\mathrm{IN}}\right) \mathrm{d} \Omega
\end{gathered}
$$

The first error is calculated in the $L_{2}$ norm in the entire domain $\Omega_{\mathrm{D}}$, whereas the second provides an assessment of the error in pressure inside the drop. Here, $\Omega_{\mathrm{IN}}$ is the part of $\Omega_{\mathrm{D}}$ for which the volume fraction of water, $F_{\mathrm{L}}$, satisfies the condition $F_{\mathrm{L}}(\mathbf{x}) \geqslant 0.99$. Correspondingly, $A_{\mathrm{D}}$ and $A_{\mathrm{IN}}$ are the areas of $\Omega_{\mathrm{D}}$ and $\Omega_{\mathrm{IN}}$.

Figure 4 shows the surface tension distributions obtained using different surface tension models; the results of the convergence study are summarized in Table III. As one can see, Modified Method B with $\alpha_{\mathrm{S}}=20^{\circ}$ has a similar convergence rate to that of Method A with $\varepsilon_{\sigma}=h$, but quite a slow convergence rate for $\alpha_{S}=10^{\circ}$. Note, however, that in terms of the $E_{2}^{P}$ error, all level set formulations display convergence slower than first order. Of all methods considered, Method B displays the slowest convergence. Comparison of the results for the error $E_{\Delta P}$ given in Table III with the corresponding values obtained by Francois et al. [32] $\left(E\left(\Delta P_{\text {total }}\right)\right.$, in their notation) shows that the level set formulations employing surface tension models Method A and Modified Method B display similar accuracy to that of the PLIC VOF method with the continuum surface force model for the surface tension. 


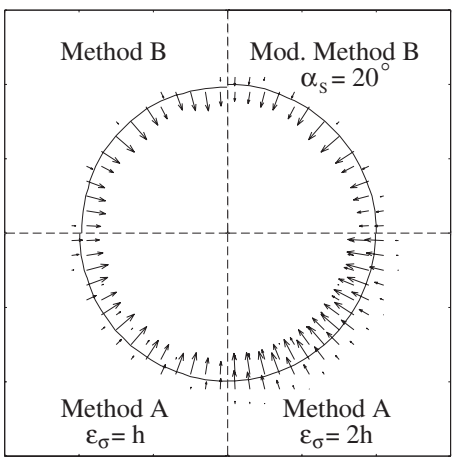

Figure 4. Surface tension distribution around the interface of a liquid drop in equilibrium; the four sectors show the results obtained using different surface tension models.

Table III. Error estimates for a liquid drop in equilibrium.

\begin{tabular}{cccccccc}
\hline & & \multicolumn{2}{c}{ Method A } & & \multicolumn{3}{c}{ Modified Method B } \\
\cline { 6 - 7 }$R / h$ & Error & $\varepsilon_{\sigma}=h$ & $\varepsilon_{\sigma}=2 h$ & Method B & $\alpha_{\mathrm{S}}=10^{\circ}$ & $\alpha_{\mathrm{S}}=15^{\circ}$ & $\alpha_{\mathrm{S}}=20^{\circ}$ \\
\hline 20 & $E_{2}^{P}$ & $7.03 \times 10^{-2}$ & $8.34 \times 10^{-2}$ & $7.33 \times 10^{-2}$ & $7.65 \times 10^{-2}$ & $7.61 \times 10^{-2}$ & $7.70 \times 10^{-2}$ \\
& $E_{\Delta P}$ & $3.94 \times 10^{-3}$ & $1.26 \times 10^{-3}$ & $6.46 \times 10^{-3}$ & $5.28 \times 10^{-3}$ & $7.43 \times 10^{-3}$ & $8.91 \times 10^{-3}$ \\
& & & & & & \\
40 & $E_{2}^{P}$ & $5.11 \times 10^{-2}$ & $6.00 \times 10^{-2}$ & $5.31 \times 10^{-2}$ & $5.39 \times 10^{-2}$ & $5.44 \times 10^{-2}$ & $5.48 \times 10^{-2}$ \\
& $E_{\Delta P}$ & $1.42 \times 10^{-3}$ & $3.59 \times 10^{-4}$ & $-6.03 \times 10^{-3}$ & $-4.62 \times 10^{-3}$ & $-4.73 \times 10^{-3}$ & $-3.81 \times 10^{-3}$ \\
\hline
\end{tabular}

\section{Zero-gravity drop oscillation}

In this example, we consider a two-dimensional planar drop oscillating around its equilibrium shape at its fundamental mode in the absence of gravity. The drop is located in an infinite, gaseous medium. According to the analytical solution obtained by Lamb [33] under the assumption of small amplitude of oscillations, the interfacial position of a drop of radius $R$, oscillating at its fundamental mode, is given in polar coordinates $(r, \theta)$, by

$$
\frac{r(\theta, t)}{R}=1+\tilde{\varepsilon} \cos (2 \theta) \cos \left(\omega_{\mathrm{d}} t\right) \exp \left(-t / \tau_{\mathrm{d}}\right)
$$

where $\tilde{\varepsilon}$ is the initial perturbation of the drop, and $\omega_{\mathrm{d}}$ and $\tau_{\mathrm{d}}$ are, respectively, the frequency and characteristic time of decay due to viscous damping:

$$
\omega_{\mathrm{d}}^{2}=\frac{6 \sigma}{R^{3}\left(\rho_{\mathrm{L}}+\rho_{\mathrm{G}}\right)}, \quad \tau_{\mathrm{d}}=\frac{R^{2}}{4} \frac{\left(\rho_{\mathrm{L}}+\rho_{\mathrm{G}}\right)}{\left(\mu_{\mathrm{L}}+3 \mu_{\mathrm{G}}\right)}
$$

The combined kinetic energy of the drop and surrounding gas is given by

$$
K_{\mathrm{T}}(t)=\frac{\pi}{4}\left(\rho_{\mathrm{L}}+\rho_{\mathrm{G}}\right) \tilde{\varepsilon}^{2} R^{2} \omega_{\mathrm{d}}^{2} \sin ^{2}\left(\omega_{\mathrm{d}} t\right) \exp \left(-2 t / \tau_{\mathrm{d}}\right)
$$


The characteristic length and velocity scales are set to $l_{\mathrm{c}}=R$ and $u_{\mathrm{c}}=\sqrt{2 \sigma /\left(R \rho_{\mathrm{L}}\right)}$, respectively. With the chosen scales, the Reynolds and Weber numbers characterizing the problem are $R e=$ $\sqrt{2 \sigma R \rho_{\mathrm{L}}} / \mu_{\mathrm{L}}$ and $W e=2$. For the numerical simulation, we choose the problem configuration analogous to those studied by other groups $[1,6,34]: \rho_{\mathrm{L}} / \rho_{\mathrm{G}}=100, \mu_{\mathrm{L}} / \mu_{\mathrm{G}}=100$, and $\operatorname{Re}=20$. The interface $r(\theta, 0)$ of the drop at $t=0$ is initialized with $\tilde{\varepsilon}=0.05$, as defined in Equation (14). See the inset in Figure 5(a) for the schematic of the initial conditions.

Owing to symmetry, it is sufficient to consider the problem in the half-domain. Thus, the computational domain is set to be $\Omega^{*}=\left\{\left(x^{*}, y^{*}\right) \mid 0 \leqslant x^{*} \leqslant 4,0 \leqslant y^{*} \leqslant 2\right\}$, with the centre of the drop located at $(2,0)$. A free-slip boundary condition is imposed along the symmetry line, and constant pressure is imposed on the rest of the domain boundary. The simulations are performed using a structured rectangular grid of resolution $200 \times 100$, with time step $\Delta t^{*}=0.01$.

Figure 5(a) shows the displacement of the interface at $\theta=\pi / 2$ as a function of time, as given by the analytical and numerical solutions. Here, Method A is employed with two different values of $\varepsilon_{\sigma}$ (namely, $h$ and $2 h$ ), and both are seen to give similar and reasonably accurate solutions. In both cases, the solution displays a clear gradual shift of the equilibrium position away from the line $r / R-1=0$, and the shift is more noticeable for the case $\varepsilon_{\sigma}=h$. We believe that this numerical artefact is caused by parasitic currents developing near the interface (to be discussed in the following section). Similarly, but less noticeably, this shift can also be seen in the solution of the axisymmetric oscillating-drop problem obtained by Sussman et al. [1] and Sussman and Smereka [34] using the ENO level set method.

In terms of the kinetic energy of the flow (Figure 5(b)), the solution obtained using Method A with $\varepsilon_{\sigma}=h$ appears to predict the dependence $K_{\mathrm{T}}^{*}\left(t^{*}\right)$ more accurately than the solution for $\varepsilon_{\sigma}=2 h$. Apparently, increasing the width of the band over which the surface tension is distributed leads to the appearance of overshoots in the values of $K_{\mathrm{T}}^{*}\left(t^{*}\right)$. This is a consequence of the asymmetry in the distribution of $\mathbf{B}$ across the interface, as the curvature, $\kappa$, is higher on the liquid side than on the gas side (see Figure 4). Reduction in the band thickness, $\varepsilon_{\sigma}$, results in a sharper resolution of $\mathbf{B}$ and a correspondingly more accurate prediction of $K_{\mathrm{T}}^{*}\left(t^{*}\right)$.

The displacement of the interface at $\theta=\pi / 2$ as a function of time obtained using Method $\mathrm{B}$ (both the original and modified formulations) is shown in Figure 6. As one can see, Method B
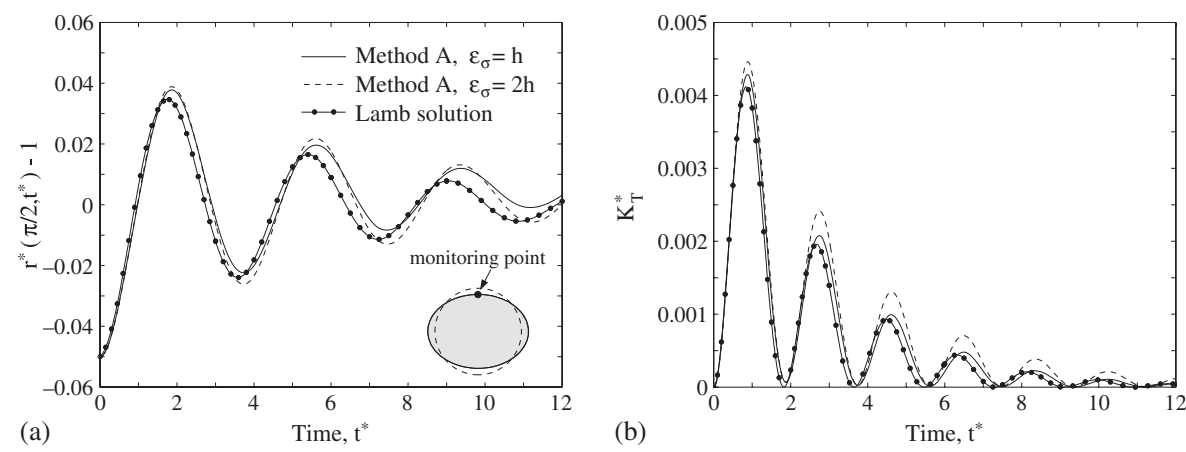

Figure 5. Simulation of a two-dimensional drop oscillating in zero gravity using Method A: (a) displacement of the interface at the monitoring point at $\theta=\pi / 2 \mathrm{vs}$ time (the inset is a schematic of the initial conditions) and (b) total kinetic energy $v s$ time. The properties of the fluid are $\rho_{\mathrm{L}} / \rho_{\mathrm{G}}=100, \mu_{\mathrm{L}} / \mu_{\mathrm{G}}=100, R e=20$, and $W e=2$. 


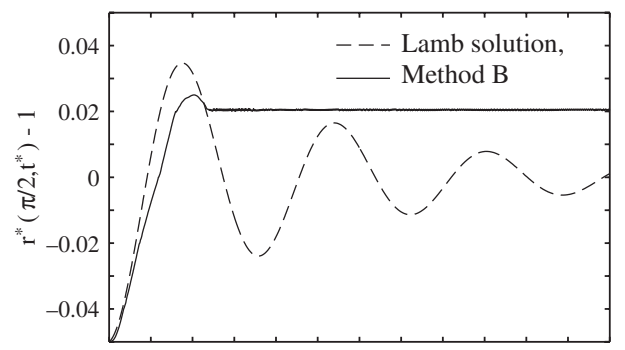

(a)

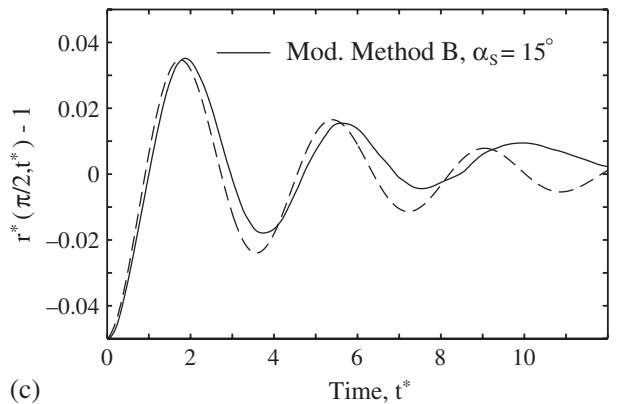

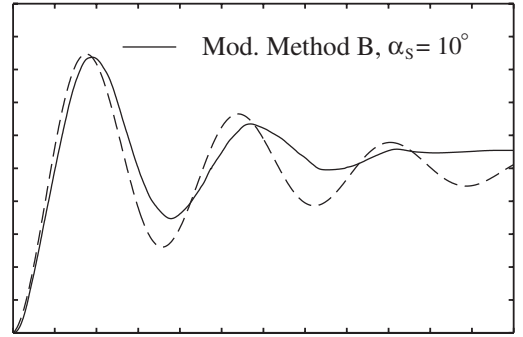

(b)

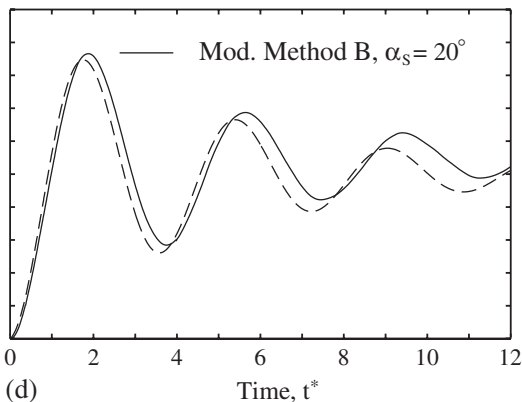

Figure 6. Oscillating two-dimensional drop in zero gravity: displacement of the interface at the monitoring point at $\theta=\pi / 2 v s$ time as predicted by (a) Method B and (b) Modified Method B with $\alpha_{\mathrm{S}}=10^{\circ}$, (c) $\alpha_{\mathrm{S}}=15^{\circ}$, and (d) $\alpha_{\mathrm{S}}=20^{\circ}$.

gives completely the wrong solution, whereas the accuracy of the solution obtained using Modified Method B depends on the value of the sector angle $\alpha_{S}$ : the larger the $\alpha_{S}$, the higher the accuracy of the solution. For instance, the value $\alpha_{\mathrm{S}}=20^{\circ}$ seems to produce a solution of comparable accuracy with that of Method A with $\varepsilon_{\sigma}=h$ (see Figure 5(a)). Figure 7 shows the kinetic energy of the flow, $K_{\mathrm{T}}^{*}$, as a function of time for Method B and Modified Method B. Evidently, of the four cases considered, only Modified Method B with $\alpha_{S}=20^{\circ}$ seems to provide good conservation of energy, whereas the other solutions display unacceptable levels of damping. The reasons for strong damping of the kinetic energy in the solutions of Modified Method $B$ for $\alpha_{S} \leqslant 15^{\circ}$ require further investigation.

As far as the mass conservation is concerned, Method B and its modified version display practically identical properties as those of Method A with $\varepsilon_{\sigma}=h$ : the mass of the drop grows almost linearly with time $M\left(t^{*}\right) \approx\left(1+\beta t^{*}\right) M_{0}$, where $\beta=6.6 \times 10^{-4}$, so that at time $t^{*}=12$ (three cycles) the relative increase in the mass of the drop is $\approx 0.8 \%$ and appears to be independent of the value of $\alpha_{S}$. Method A with $\varepsilon_{\sigma}=2 h$ shows better mass conservation with $\beta=3.3 \times 10^{-4}$.

\section{Parasitic currents}

Parasitic currents are unphysical flows developing around the interface as a result of a local imbalance of the pressure and stresses in the interfacial region caused by discretization errors. These currents are known to have an adverse effect on the performance of numerical interface-tracking methods, particularly when the flow configuration is close to equilibrium [9]. 

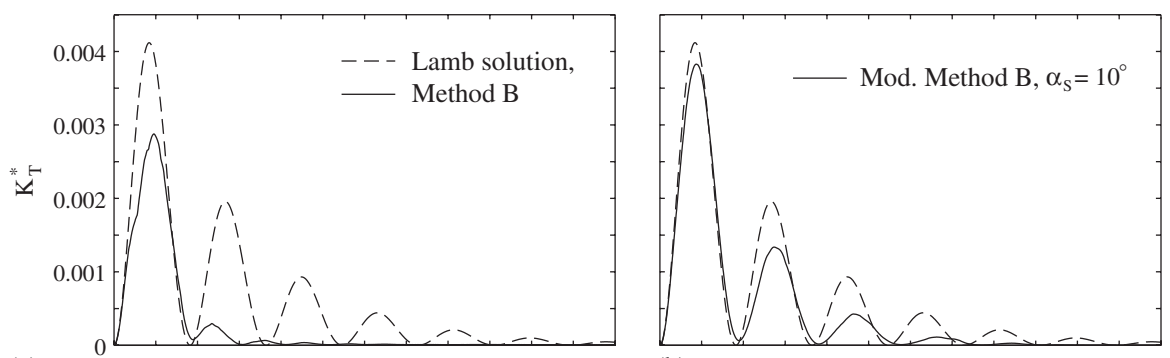

(a)

(b)
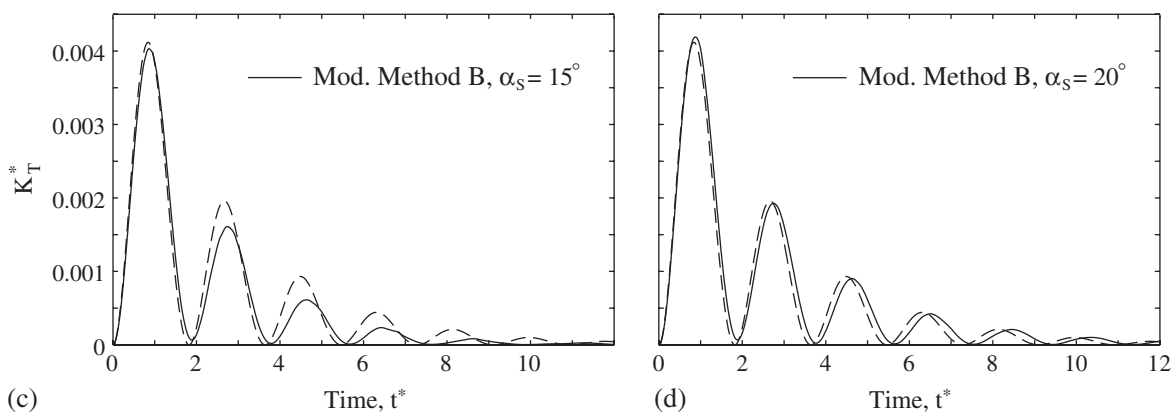

Figure 7. Oscillating two-dimensional drop in zero gravity: total kinetic energy of the flow $v s$ time as predicted by (a) Method B and (b) Modified Method B with $\alpha_{S}=10^{\circ}$, (c) $\alpha_{S}=15^{\circ}$, and (d) $\alpha_{S}=20^{\circ}$.

In order to compare the intensities of the parasitic flows developing for the two surface tension models, Methods A and B, we simulate a static planar drop of liquid with zero initial disturbance. Theoretically, if such a drop is placed in a stagnant gaseous medium in the absence of gravity, the drop and the surrounding medium should remain motionless. In a numerical simulation, however, this does not happen, due to the developing parasitic currents. Consequently, it becomes important to control and minimize their amplitude and rate of growth. For the simulation, we use the problem configuration identical to that used in the previous section for the oscillating drop, except that the initial disturbance, $\tilde{\varepsilon}$, is set to 0 . The problem of parasitic currents has received much attention in the literature, and a liquid drop of similar configuration has been studied in $[5,6,9]$.

Figure 8 shows the total kinetic energy of the parasitic currents defined as $K_{P C}^{*}=\int_{\Omega^{*}} \frac{1}{2} \rho^{*} u^{* 2} \mathrm{~d} \Omega^{*}$ $v s$ time for $t^{*} \leqslant 12$. We note that for the solutions obtained using Method $\mathrm{A}$, the scale of $K_{P C}^{*}$ is approximately the same for $\varepsilon_{\sigma}=h$ and $2 h$, with values slightly lower for $\varepsilon_{\sigma}=2 h$. The intensity of the parasitic currents, as measured by $K_{P C}^{*}$, observed in simulations carried out using Modified Method B appears to depend on the value of $\alpha_{S}$ employed. The value $\alpha_{S}=20^{\circ}$ yields the lowest average level of the $K_{P C}^{*}$, which is close to that produced using Method A with $\varepsilon_{\sigma}=2 h$. Method B gives the highest (by one order of magnitude) value of the $K_{P C}^{*}$.

Another common measure of the intensity of the parasitic currents is the maximum velocity $u_{\max }^{*}$ observed in the computational domain during a simulation $[9,10]$. We found in this example that the value of $u_{\max }^{*}\left(t^{*}\right)$ produced using Method A with $\varepsilon_{\sigma}=2 h$ and $h$ rapidly reach the maximum values 0.028 and 0.02 , respectively, and afterwards practically do not change with time. The functions $u_{\max }^{*}\left(t^{*}\right)$ for Modified Method $\mathrm{B}$ oscillate slightly around the average value of $\approx 0.03$ 


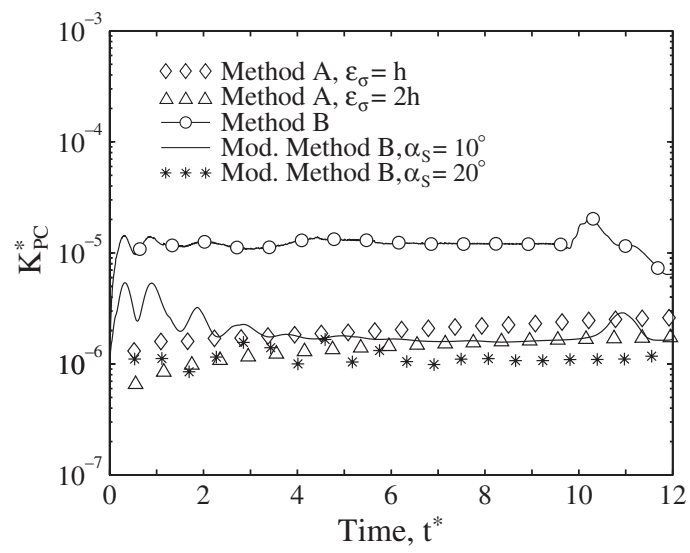

Figure 8. Kinetic energy of parasitic currents in the problem of the two-dimensional static drop in zero gravity of zero initial disturbance. The properties of the fluids are $\rho_{\mathrm{L}} / \rho_{\mathrm{G}}=100$, $\mu_{\mathrm{L}} / \mu_{\mathrm{G}}=100, R e=20$, and $W e=2$.

for $\alpha_{\mathrm{S}}=10^{\circ}, 15^{\circ}$, and $20^{\circ}$. For Method $\mathrm{B}$, the dependence of $u_{\max }^{*}$ on time is highly oscillatory with the average value equal to 0.1 and local maxima as high as 0.22 .

A more detailed analysis of the parasitic currents generated by the FE-FV level set method is given in [26]. From the results obtained there, together with those given here, we can summarize that in general the magnitudes of the parasitic velocities of the FE-FV level set method fall in the same range as those of the second-order PLIC VOF algorithms [9, 10].

As a last comment to this section, we note that the average and maximum velocities of the parasitic flow produced by the PLIC VOF method have been reported to be proportional to the ratio $\sigma / \mu_{\mathrm{L}}[9,10]$. The Reynolds number defined in terms of this parasitic velocity is equal to the Laplace number, $L a=\sigma \rho_{\mathrm{L}} l_{\mathrm{c}} / \mu_{\mathrm{L}}^{2}$, which is a non-dimensional number characterizing the dissipation processes in the interfacial region. Owing to the similarity of the ways in which the surface tension force, phase densities, and viscosities are interpolated across the interface by the level set or PLIC VOF methods, it is reasonable to assume that the intensity of the parasitic currents produced by the level set method is also proportional to the value of $L a$, although we cannot confirm this at the moment. Nevertheless, for reference purposes, we provide here, and in the following two sections, the characteristic values of the $L a$ number. In the present application of the zero-gravity drop oscillation, $L a=200$.

\section{Gas bubble rising in a viscous fluid}

The next application is of an axisymmetric air bubble rising in a viscous fluid. For this simulation, we use data from the experiment of Hnat and Buckmaster [30], who examined terminal topologies of air bubbles in mineral oil. The density and viscosity of the oil are $\rho_{\mathrm{L}}=876 \mathrm{~kg} / \mathrm{m}^{3}$ and $\mu_{\mathrm{L}}=$ $0.118 \mathrm{~kg} / \mathrm{ms}$, giving oil /air density and viscosity ratios as follows: $\rho_{\mathrm{L}} / \rho_{\mathrm{G}}=714$ and $\mu_{\mathrm{L}} / \mu_{\mathrm{G}}=6670$; the surface tension is $\sigma=0.032 \mathrm{~N} / \mathrm{m}$. In their experiment, Hnat and Buckmaster studied bubbles of different volumes, whereas here we model only one bubble: that of the smallest volume $\left(V_{\mathrm{b}}=\right.$ $\left.0.94 \mathrm{~cm}^{3}\right)$, and correspondingly the lowest rise velocity $\left(U_{\mathrm{t}}^{\exp }=21.5 \mathrm{~cm} / \mathrm{s}\right)$, of those considered. 
For this case, the surface tension effects are the strongest in comparison with buoyancy, so that it represents the most challenging validation test for a surface tension model.

Initially, at $t=0$, the bubble is approximated as a sphere of radius $r_{\mathrm{b}}=0.61 \mathrm{~cm}$. Setting the distance and velocity scales to $l_{\mathrm{c}}=r_{\mathrm{b}}$ and $u_{\mathrm{c}}=U_{\mathrm{t}}^{\mathrm{exp}}$, respectively, gives the following dimensionless groups: $R e=9.8, F r=0.76, W e=7.6$, and $L a=12.3$. As the problem is axisymmetric, only half of the bubble is modelled, the simulation being carried out in cylindrical coordinates on the computational domain $\Omega^{*}\left(r^{*}, z^{*}\right)$ of size $5.5 \times 20$ (width $\times$ height). At the sides of the fluid domain, $\Omega^{*}$, except for the symmetry line at $r^{*}=0$, the fluid pressure is set to the hydrostatic value $p=\rho_{\mathrm{L}} g z$. The centre of the bubble is initially at $\left(r^{*}, z^{*}\right)=(0,4)$.

Calculations have been performed with mesh resolution $h^{*}=0.044$ and time step $\Delta t^{*}=0.01$. As was shown in [26], the thickness of the velocity boundary layer developing on the surface of this bubble is estimated to be about $0.43 r_{\mathrm{b}}$, so that the chosen mesh resolution is adequate for the problem under consideration. Surprisingly, all level set formulations used for this simulation displayed robust convergence and predicted almost identical bubble shapes. This can be seen in Figure 9, which shows the bubble cross-sections at different times for each model employed. According to the simulations, the bubble reaches terminal velocity after $t^{*}=8$, corresponding to a physical time of $0.22 \mathrm{~s}$. The terminal bubble rise velocities and aspect ratios (the ratio of the bubble width to its height) are given in Table IV and compared with the measured values. Clearly, there is good agreement for all surface tension models. In addition, Figure 10(a) shows the position of the centre of mass of the bubble $v s$ time obtained using Method A (with $\varepsilon_{\sigma}=2 h$ ) and Method B. The velocity field inside and outside of the bubbles at $t=0.27 \mathrm{~s}$ is shown in Figures 10 (b) and (c). (The other two surface tension models predict almost the same results, and for this reason are not shown.)

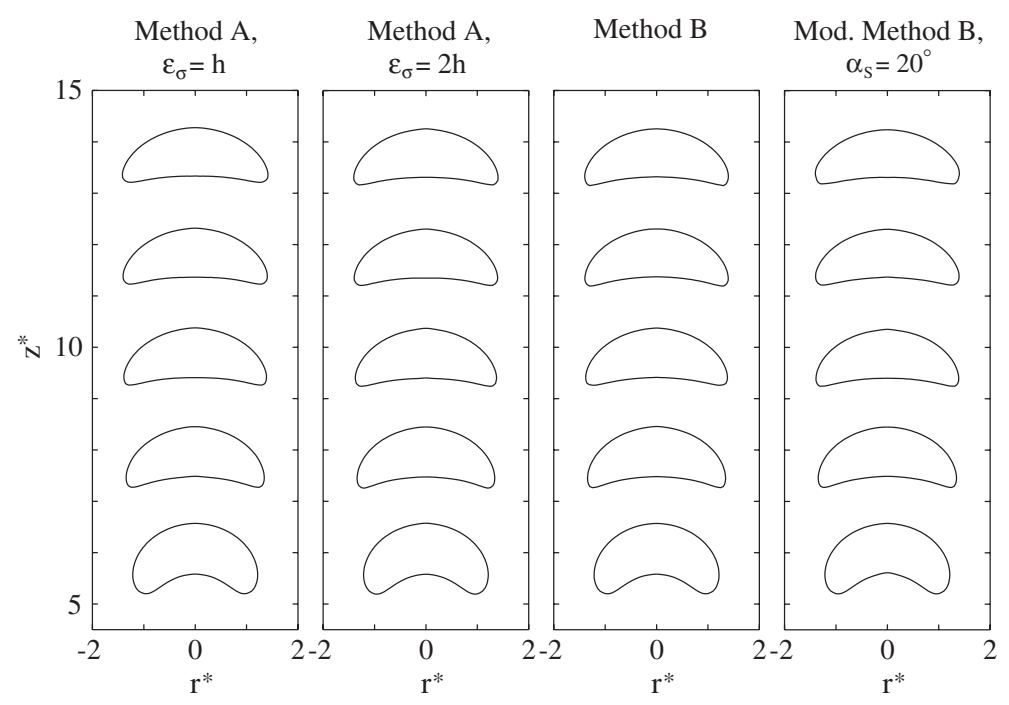

Figure 9. Cross-sections of an air bubble rising in mineral oil, as predicted using Method A, $\varepsilon_{\sigma}=h$; Method A, $\varepsilon_{\sigma}=2 h$; Method B; Modified Method B, $\alpha_{\mathrm{S}}=20^{\circ}$; at times $t^{*}=2.0,4.0,6.0,8.0,10.0$. Initially, the bubble is a sphere of radius $r^{*}=1$, with the centre at $z^{*}=4$. 
Table IV. Terminal rise velocities, $U_{\mathrm{t}}$, and aspect ratios, $w_{\mathrm{b}} / l_{\mathrm{b}}$, of a fully developed spherical-cap air bubble in oil; experimental data from Hnat and Buckmaster [30].

\begin{tabular}{|c|c|c|c|c|c|}
\hline \multirow[b]{2}{*}{ Results } & \multirow[b]{2}{*}{ Experiment } & \multicolumn{2}{|c|}{ Method A } & \multirow[b]{2}{*}{ Method B } & \multirow{2}{*}{$\begin{array}{l}\text { Modified Method B } \\
\alpha_{S}=20^{\circ}\end{array}$} \\
\hline & & $\varepsilon_{\sigma}=h$ & $\varepsilon_{\sigma}=2 h$ & & \\
\hline$U_{\mathrm{t}}(\mathrm{cm} / \mathrm{s})$ & 21.5 & 21.2 & 21.1 & 21.1 & 21.3 \\
\hline$w_{\mathrm{b}} / l_{\mathrm{b}}$ & 2.7 & 2.7 & 2.6 & 2.6 & 2.7 \\
\hline
\end{tabular}
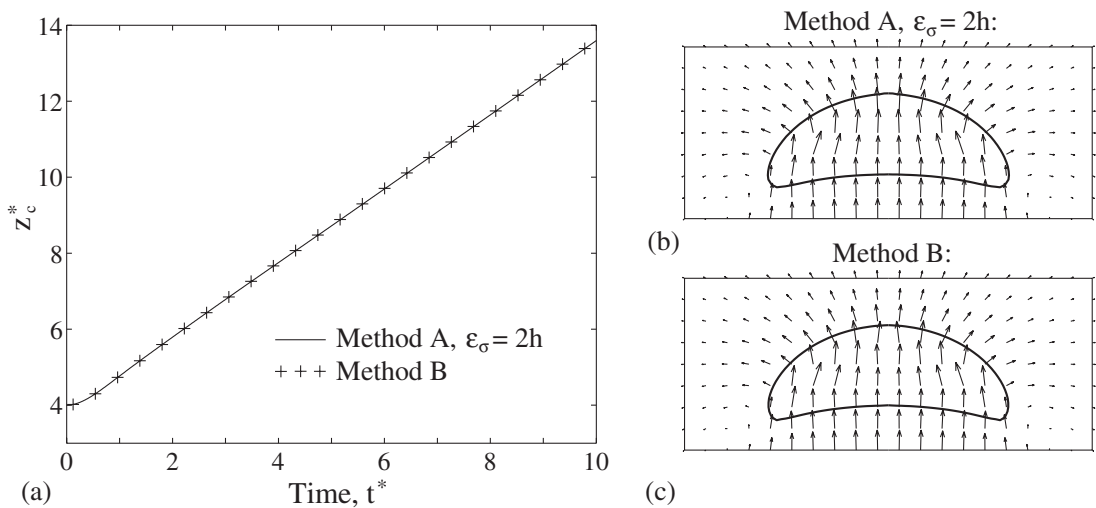

(b)

Method B:

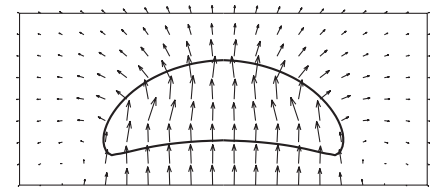

(c)

Figure 10. Solution of the problem of an air bubble rising in mineral oil using Method A, $\varepsilon_{\sigma}=2 h$ and Method B: (a) position of the centre of mass of the bubble $z_{\mathrm{c}}^{*} v s$ time, and (b) velocity field inside and outside of the bubble at $t^{*}=10$.

A convergence study is performed by successively refining the mesh size $h^{*}: 0.1,0.067,0.044$ (the mesh is refined using a ratio of $\frac{2}{3}$, rather than $\frac{1}{2}$, due to the limited computer resources available). The difference in solutions at $t^{*}=10$ obtained for successive mesh resolutions is defined as follows:

$$
E_{1}^{i}=\frac{2}{\pi r_{\mathrm{b}}^{2}} \int_{\Omega}\left|H\left(\phi_{\mathrm{f}}(\mathbf{x})\right)-H\left(\phi_{\mathrm{c}}(\mathbf{x})\right)\right| \mathrm{d} \Omega
$$

where $\phi_{\mathrm{f}}$ is the solution using a refined mesh and $\phi_{\mathrm{c}}$ is the solution from a coarser mesh. The results given in Table $\mathrm{V}$ show that for this particular application the convergence rate of Method A appears to be $O\left(h^{3 / 2}\right)$, whereas those of Method B and Modified Method B are close to first order. Mass conservation for Modified Method B appears to be slightly better than for Method A, as can be seen in Figure 11. However, the results for the simulation of the $2 \mathrm{D}$ oscillating drop indicate that this is not a general trend.

\section{Coalescence of two oil droplets}

The final application is of the merging of two droplets under the effect of surface tension into a single drop. The purpose of this example is to demonstrate the ability of Method B and Modified Method B to handle interfaces of changing topology and to compare their predictions with those of Method A. The model consists of two two-dimensional drops of oil in air in the absence of 
Table V. Error estimate $E_{1}^{i}$ at time $t^{*}=10$ for the simulation of an air bubble rising in oil.

\begin{tabular}{lccc}
\hline$h^{*}$ & Method A & Method B & $\begin{array}{c}\text { Modified method B } \\
\left(\alpha_{\mathrm{S}}=20^{\circ}\right)\end{array}$ \\
\hline 0.1 & N/A & N/A & N/A \\
0.067 & 0.31 & 0.34 & 0.32 \\
0.044 & 0.16 & 0.20 & 0.20
\end{tabular}

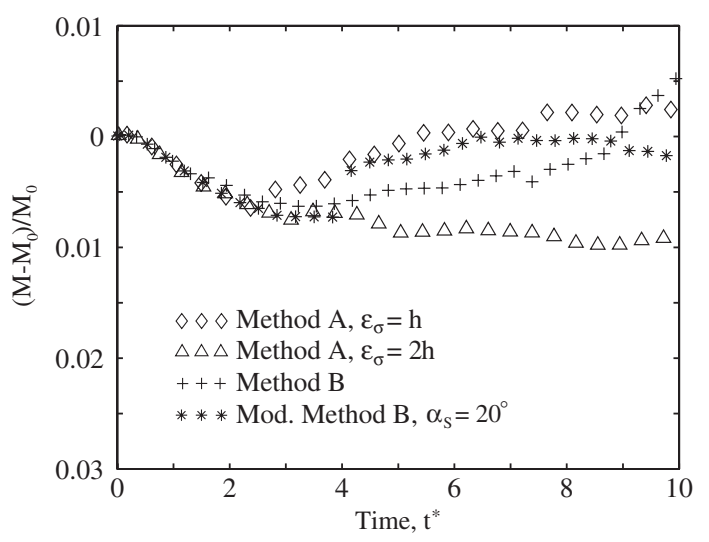

Figure 11. Mass of the bubble as a function of time in the solution of the problem of the air bubble rising in mineral oil; $M$ is the mass of air contained inside the bubble and $M_{0}=M$ at $t=0$.

gravity (see Figure 12). Initially, the drops are of elliptic shape: the larger drop has semi-axes $a_{1}=1.41 \mathrm{~cm}$ and $b_{1}=0.71 \mathrm{~cm}$, and the smaller drop $a_{2}=0.82 \mathrm{~cm}$ and $b_{2}=0.55 \mathrm{~cm}$. The surface tension coefficient, densities, and viscosities of the oil and air are set identical to those used in the simulation of the rising air bubble of the previous section. The distance and velocity scales are set to $l_{\mathrm{c}}=a_{1}$ and $u_{\mathrm{c}}=\sqrt{2 \sigma /\left(a_{1} \rho_{\mathrm{L}}\right)}$, respectively. With this scaling, the non-dimensional groups are $R e=7.5, W e=2$, and $L a=28.4$.

Owing to symmetry, the problem is solved in the half-domain $\Omega^{*}=\left\{\left(x^{*}, y^{*}\right) \mid-1.5 \leqslant x^{*} \leqslant 1.5\right.$, $\left.0 \leqslant y^{*} \leqslant 1.5\right\}$. On the symmetry line $y^{*}=0$, a free-slip condition is imposed, and on the rest of the domain boundaries the pressure is set to $p^{*}=0$. A uniform computational grid with $h^{*}=0.05$ is employed. The initial distance between the drops is set to the small value $1.2 h^{*}$ to ensure that the merging of the drops occurs at an early phase in the transient. At time $t^{*}=0$, the centre of gravity of the system is at $x^{*}=0$. The value of the time step is set to $\Delta t^{*}=4.5 \times 10^{-3}$, which is equal to $0.9 \Delta t_{\text {cap }}^{*}$ with $\Delta t_{\text {cap }}$ being the capillary time step restriction [8]: $\Delta t_{\text {cap }}=\left[\left(\rho_{\mathrm{L}}+\rho_{\mathrm{G}}\right) h^{3} /(4 \pi \sigma)\right]^{1 / 2}$. The simulations are carried out using Method B, Modified Method B with $\alpha_{S}=20^{\circ}$, and Method A with $\varepsilon_{\sigma}=h$, as these choices yield the sharpest resolution of the surface tension.

The interfaces at progressive times, as predicted by the three methods, are shown in Figure 12. Method B and Modified Method B produce very similar results: the difference in interface shapes obtained using the two methods is discernable only on the last snapshot. The results obtained using Method A are similar, though a slightly faster development of the interface may be discerned 


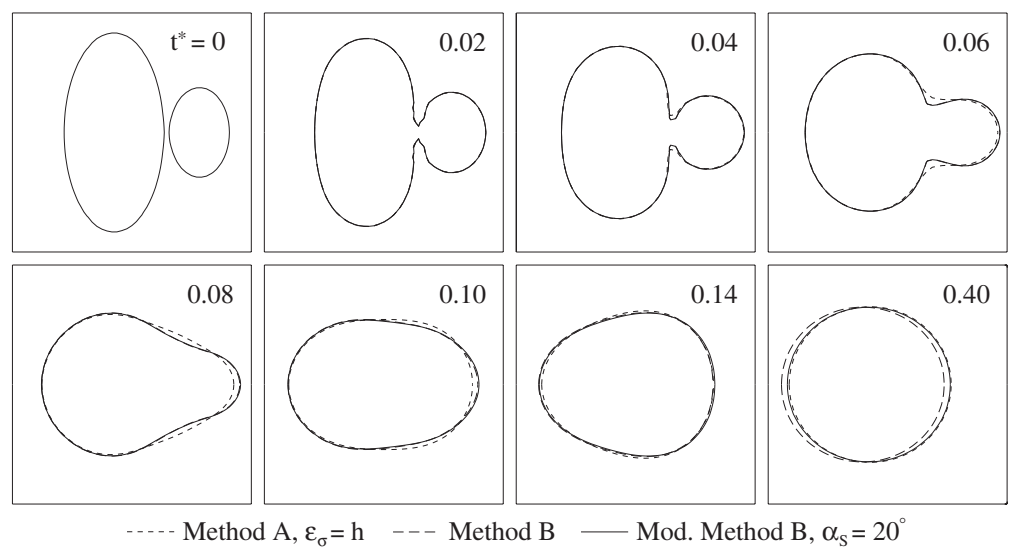

Figure 12. Coalescence of oil droplets in zero gravity: interface development for different surface tension models $\left(t^{*}=t / t_{\mathrm{c}}, t_{\mathrm{c}}=0.2 \mathrm{~s}\right)$.
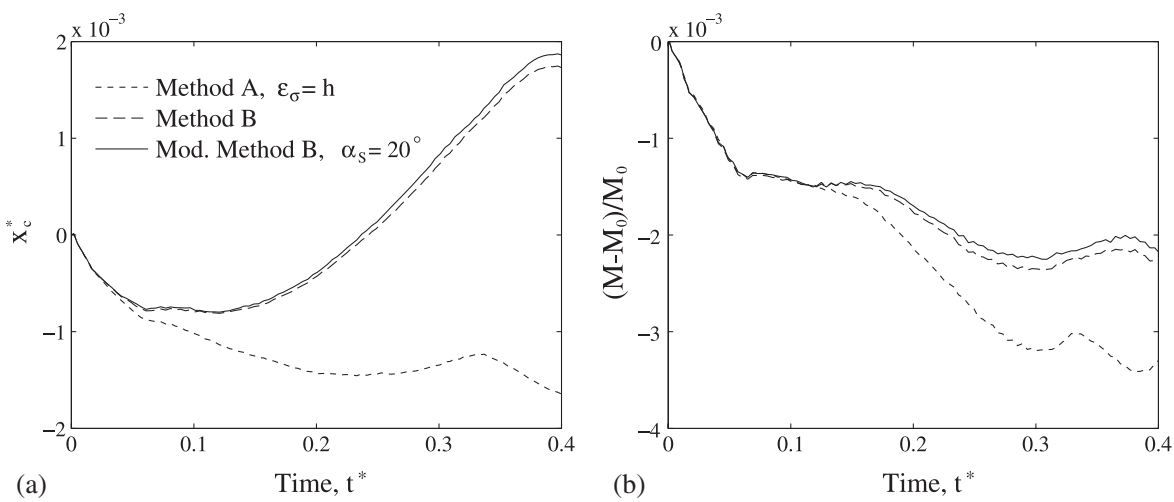

Figure 13. Coalescence of oil droplets in zero gravity: (a) position of the centre of gravity of the oil $x_{\mathrm{c}}^{*}$ $v s$ time and (b) mass of oil $v s$ time, where $M_{0}$ is the mass of oil at $t=0$.

starting when the drops first merge together. By $t^{*}=0.4$, the newly formed drop is approaching its final, spherical equilibrium state.

As there are no external forces on the system, the centre of gravity should not change as the two drops coalesce. The coordinate of the centre of gravity of the oil, $x_{\mathrm{c}}^{*}$, as a function of time is shown in Figure 13(a). The three surface tension models employed here display similar accuracy: by the end of the simulation, at $t^{*}=0.4$, the centre of gravity in each case is displaced by a small quantity $\approx 2 \times 10^{-3}$, which is within the numerical tolerance. Mass conservation is good in all cases, though slightly improved for Method B and Modified Method B than for Method A (Figure 13(b)). Apparently, in this simulation, the value of the integration error in $\mathbf{B}$ produced by Method A is very small, which is a consequence of the fact that the contact area of the droplet interfaces during coalescence is very small (see Appendix B). 


\section{CONCLUSIONS}

A comparative study of two surface tension models for simulating two-phase interfacial flows using the level set interface-tracking method has been undertaken. Both models are incorporated into a hybrid finite-element/finite-volume (FE-FV) level set formulation comprising a FE treatment of the level set equations and a FV solution of the Navier-Stokes equations. Earlier, we had implemented the FE-FV level set method into the commercial CFD code CFX-4, and this modified code was employed in this study.

The first surface tension model (referred to in the text as Method A) is based on the traditional approach in which the force is distributed in a band around the interface using a regularized delta function. In the second model (referred to as Modified Method B), the force is partly distributed in a band around the interface and partly localized to the computational cells actually containing the interface. With the prerequisite of supplying an adequate mesh resolution, the traditional surface tension model, Method A, is generally superior to Modified Method B in terms of accuracy. However, we have demonstrated that in the particular case of merging and pinching-off of interfaces the traditional surface tension model, Model A, produces an error that results in non-converging solutions for interfaces with large contact area (film-like interfaces) due to overlapping of the surface tension bands. In contrast, Modified Method B, although only first-order accurate, displays consistent convergence for all cases considered, including poorly resolved film-like interfaces.

For applications not involving film-like interfaces, level set formulations employing Method A and Modified Method B produce results of similar accuracy. In these applications, the convergence rate of the solutions obtained using Model A has been found to vary between $O(h)$ and $O\left(h^{3 / 2}\right)$, where $h$ is the mesh size, instead of the expected second-order convergence rate. The main reason for the observed lack of accuracy is shown to be the smoothing of the density across the interface using regularized Heaviside functions, which reduces the formal accuracy of the solution to first order, and obscures the benefits obtained from more accurate surface tension modelling.

The results obtained imply that for the case of merging and pinching-off interfaces with large contact area, Modified Method B can produce more accurate solutions than the traditional level set approach based on Method A. Possibly, the most efficient approach would be to combine both surface tension models into a single formulation using Method A on the well-resolved parts of the interface and Modified Method B on the rest. Such a hybrid model would then take the best advantage of both models.

\section{APPENDIX A}

Tornberg and Engquist [18] have shown that, for a one-dimensional delta function, $\delta_{\varepsilon}(x)$, to be of order $q$, which implies that the integration error $E$ satisfies the following inequality

$$
E=\left|h \sum_{j=-\infty}^{\infty} \delta_{\varepsilon}\left(x_{j}-\bar{x}\right) f\left(x_{j}\right)-f(\bar{x})\right| \leqslant C h^{q}
$$

for any function $f(x) \in C^{q}(\Re)$ with $C$ being a constant and $q$ an integer, the function $\delta_{\varepsilon}(x)$ must have compact support $[-\varepsilon, \varepsilon]$ with $2 \varepsilon \geqslant q h$, and its discrete moments $M_{r}=$ $\sum_{j=-\infty}^{\infty} \delta_{\varepsilon}\left(x_{j}-\bar{x}\right)\left(x_{j}-\bar{x}\right)^{r}$ must be equal to 1 for $r=0$, and 0 for all $r=1, \ldots, q-1$. 
Evidently, the sharpest resolution of the surface tension force via $\delta_{\varepsilon}(\phi)$ is achieved with $\varepsilon=h$, which allows the maximum achievable convergence rate of $q=2$. The three functions $\delta_{\varepsilon}(\phi)$ given below are the ones most often used.

(a) As in the original work of Sussman et al. [1], the traditional choice for $\delta_{\varepsilon}(\phi)$ in level set formulations has been the cosine delta function:

$$
\delta_{\varepsilon}^{\cos }(\phi)= \begin{cases}\frac{1}{2 \varepsilon}(1+\cos (\pi \phi / \varepsilon)), & |\phi / \varepsilon| \leqslant 1 \\ 0, & |\phi / \varepsilon|>1\end{cases}
$$

which, being formally only first-order accurate $(q=1)$, in practice normally displays convergence close to second order (see [18] for an explanation).

(b) The simplest delta function constructed from polynomials is the linear hat delta function

$$
\delta_{\varepsilon}^{\mathrm{L}}(\phi)=\left\{\begin{array}{l}
\frac{1}{\varepsilon}(1-|\phi / \varepsilon|), \quad|\phi / \varepsilon| \leqslant 1 \\
0, \quad|\phi / \varepsilon|>1
\end{array}\right.
$$

which is formally second-order accurate, i.e. $q=2[18,19]$.

(c) Recently, Tornberg and Engquist [18] and Engquist et al. [19] demonstrated that integration over a contour using a one-dimensional delta function with constant support, i.e. $\varepsilon=$ const, can produce $O(1)$ errors if the contour has straight extended segments. This lack of accuracy is observed, for instance, with functions defined by Equations (A2) and (A3). On curved contours without straight segments, the local integration errors are of different signs, so that they cancel each other out and the overall accuracy of integration is apparently restored. Nevertheless, in order to ensure consistent convergence, Engquist et al. [19] proposed the following modified linear hat regularized delta function that results in first-order convergence regardless of the shape of the contour:

$$
\delta_{\varepsilon\left(\nabla \phi, \varepsilon_{0}\right)}^{\mathrm{L}}(\phi)=\left\{\begin{array}{l}
\frac{1}{\varepsilon}(1-|\phi / \varepsilon|), \quad|\phi / \varepsilon| \leqslant 1 \\
0, \quad|\phi / \varepsilon|>1
\end{array}, \quad \varepsilon=\varepsilon_{0}|\nabla \phi|_{1} /|\nabla \phi|\right.
$$

where $|\nabla \phi|_{1}=\left|\phi_{x}\right|+\left|\phi_{y}\right|+\left|\phi_{z}\right|$.

\section{APPENDIX B}

In this appendix, we derive an estimate of the error produced by Method A with $\varepsilon_{\sigma}=h$ for film-like interfaces due to overlapping surface tension bands. First, consider a one-dimensional analogy of the problem. The grid is uniform with cell size $h$. Let an interface be located at point $x=x_{I}$. Then, in the general case, the distributed surface tension force near the interface will consist of two contributions $B_{i}, i=1,2$, defined at the two FV nodes located in the interval $\left[x_{I}-h, x_{I}+h\right]$. Accordingly, if there are two interfaces, accurate representation of the surface tension requires four contributions $B_{i}, i=1, \ldots, 4$. However, if the two interfaces approach each other within a distance $\Delta R$, where $h \leqslant \Delta R \leqslant 2 h$, in such a way that there is only one node between them, as this node cannot contain two values of $\phi_{i}$, one surface tension contribution at this node corresponding 
to the larger value of $\left|\phi_{i}\right|$ will be lost. Ultimately, this situation results in underestimation of the net surface tension force.

Consider this particular case in detail. The net surface tension force in the one-dimensional case is given by $B=\sum_{i=1,4} h B_{i}$. Denote the lost contribution that is not included in the summation by $B_{j}$. Analysis shows that the value of $B_{j}$ can vary between 0 and $\sigma \kappa \delta_{h}(\Delta R / 2)$. Taking the linear hat delta function for simplicity, the average value of $B_{j}$ is given by $\bar{B}_{j}=\sigma \kappa \delta_{h}^{\mathrm{L}}(\Delta R / 2) / 2$. Simplifying, one obtains $\bar{B}_{j}=\sigma \kappa(2 h-\Delta R) / 4 h^{2}$. The probability, $p$, of having only one node between two interfaces located within the distance $\Delta R$ from each other is equal to $(2 h-\Delta R) / h$. The average error $\bar{E}_{\mathrm{ST}}^{1 D}$ in the value of $B$ in the one-dimensional case is then equal to $h p \bar{B}_{j}$, so that finally we obtain

$$
\bar{E}_{\mathrm{ST}}^{1 D} \approx \sigma \kappa(2-\Delta R / h)^{2} / 4
$$

In the two-dimensional case, the overall error in the value of $\mathbf{B}$ can be assumed to be the sum of the local errors $h^{2} B_{j} \mathbf{n}_{j}$ produced at the nodes in the region between the interfaces. Consequently, the average error in $\mathbf{B}$ for a two-dimensional film-like interface is

$$
\overline{\mathbf{E}}_{\mathrm{ST}} \approx \frac{\sigma(2-\Delta R / h)^{2}}{4} \int_{\gamma_{\mathrm{m}}} \kappa \mathbf{n} \mathrm{d} \gamma
$$

where the integral is taken over the curve $\gamma_{\mathrm{m}}$ midway between the two interfaces. In the twodimensional case, the integral can be evaluated as the difference in tangent unit vectors, $\Delta \gamma_{m}$, using the Frenet-Serret formula. In the three-dimensional case, the integration error is also given by Equation (B2), but with $\gamma_{\mathrm{m}}$ being the contact area of the interfaces.

Analogously, Method A produces an error in the case of colliding and merging drops, though, in contrast to the film-like interfaces, this situation does not result in a non-converging solution. To see this, consider two merging two-dimensional drops of the same radius $R$. As one can see from Figure B1, the height of the contact region of the surface tension bands in this application is $A=O(\sqrt{R h})$. Using Equation (B2), we obtain that the integration error in the value of $B$ in this case is of order $O(\sigma \kappa \sqrt{R h})$, which, taking into account that $\kappa \approx 1 / R$, gives an error of order $O(\sigma \sqrt{h / R})$. Generalizing to three dimensions, the area of the contact region is $O(R h)$, and the integration error is $O(\sigma h)$. Hence, in the case of colliding and merging drops, the error converges to zero as the grid is refined.
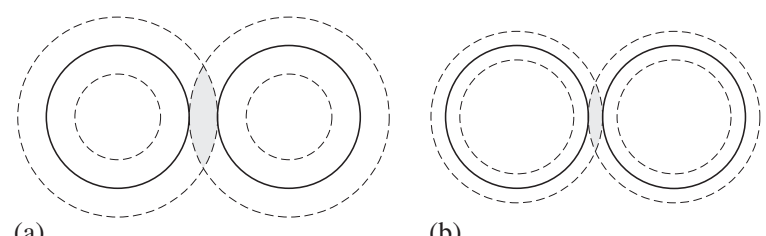

(b)

Figure B1. Schematic of two colliding droplets of the same radius: in case (a) the mesh size, $h$, is double that of case (b). The solid line shows the droplet surfaces. The dashed lines show the level sets $\phi=\varepsilon_{\sigma}$ and $\phi=-\varepsilon_{\sigma}$, which mark the boundaries of the bands over which the surface tension force in Method A is distributed. 


\section{REFERENCES}

1. Sussman M, Smereka P, Osher S. A level set approach for computing solutions to incompressible two-phase flow. Journal of Computational Physics 1994; 114:146-159.

2. Sethian JA. Level Set Methods and Fast Marching Methods: Evolving Interfaces in Computational Geometry, Fluid Mechanics, Computer Vision, and Materials Science. Cambridge University Press: Cambridge, U.K., 1999.

3. Sussman M, Fatemi E. An efficient interface-preserving level set redistancing algorithm and its application to interfacial incompressible fluid flow. SIAM Journal on Scientific Computing 1999; 20:1165-1191.

4. Losasso F, Fedkiw R, Osher S. Spatially adaptive techniques for level set methods and incompressible flow. Computers and Fluids 2006; 35:995-1010.

5. Popinet S, Zaleski S. A front-tracking algorithm for accurate representation of surface tension. International Journal for Numerical Methods in Fluids 1999; 30:775-793.

6. Tryggvason G, Bunner B, Esmaeeli A, Juric D, Al-Rawahi N, Tauber W, Han J, Nas S, Jan Y-J. A front-tracking method for the computations of multiphase flow. Journal of Computational Physics 2001; 169:708-759.

7. Hou TY, Lowengrub JS, Shelley MJ. Boundary integral methods for multicomponent fluids and multiphase materials. Journal of Computational Physics 2001; 169:302-323.

8. Brackbill JU, Kothe DB, Zemach C. A continuum method for modeling surface tension. Journal of Computational Physics 1992; 100:335-354.

9. Lafaurie B, Nardone C, Scardovelli R, Zaleski S, Zanetti G. Modelling merging and fragmentation in multiphase flows with SURFER. Journal of Computational Physics 1994; 113:134-147.

10. Meier M, Yadigaroglu G, Smith BL. A novel technique for including surface tension in PLIC-VOF methods. European Journal of Mechanics B-Fluids 2002; 21:61-73.

11. Tryggvason G, Bunner B, Esmaeeli A, Al-Rawahi N. Computations of multiphase flows. Advances in Applied Mechanics 2003; 39:81-120.

12. Kothe DB. Perspective on Eulerian finite volume methods for incompressible interfacial flows. Technical Report LA-UR-97-3559, Los Alamos National Laboratory, Los Alamos, NM 87545, U.S.A., 1999.

13. Liu X-D, Fedkiw RP, Kang M. A boundary condition capturing method for Poisson's equation on irregular domains. Journal of Computational Physics 2000; 160:151-178.

14. Smereka P. The numerical approximation of a delta function with application to level set methods. Journal of Computational Physics 2006; 211:77-90.

15. Kang M, Fedkiw RP, Liu X-D. A boundary condition capturing method for multiphase incompressible flow. Journal of Scientific Computing 2000; 15:323-360.

16. Hong J-M, Shinar T, Kang M, Fedkiw RP. On boundary condition capturing for multiphase interfaces. Journal of Scientific Computing 2007; 31:99-125.

17. Tornberg A-K, Engquist B. Regularization techniques for numerical approximation of PDEs with singularities. Journal of Scientific Computing 2003; 19:527-552.

18. Tornberg A-K, Engquist B. Numerical approximations of singular source terms in differential equations. Journal of Computational Physics 2004; 200:462-488.

19. Engquist B, Tornberg A-K, Tsai R. Discretization of Dirac delta functions in level set method. Journal of Computational Physics 2005; 207:28-51.

20. Ilic M, Wörner M, Cacuci DG. Balance of liquid-phase turbulence kinetic energy equation for bubble-train flow. Journal of Nuclear Science and Technology 2004; 41:331-338.

21. CFX-4, User Documentation. AEA Technology, Harwell: Oxfordshire, U.K., 2000.

22. Kikugawa G, Takagi S, Matsumoto Y. A molecular dynamics study on liquid-vapor interface adsorbed by impurities. Computers and Fluids 2007; 36:69-76.

23. Barth TJ, Sethian JA. Numerical schemes for the Hamilton-Jacobi and level set equations on triangulated domains. Journal of Computational Physics 1998; 145:1-40.

24. Van Doormaal JP, Raithby GD. Enhancements of the SIMPLE method for predicting incompressible fluid flows. Numerical Heat Transfer 1984; 7:147-163.

25. Shepel SV, Smith BL, Paolucci S. Implementation of a level set interface tracking method in the FIDAP and CFX-4 codes. Journal of Fluids Engineering-Transactions of the ASME 2005; 127:674-686.

26. Shepel SV, Smith BL. New finite-element/finite-volume level set formulation for modelling two-phase incompressible flows. Journal of Computational Physics 2006; 218:479-494.

27. Rudman M. A volume-tracking method for incompressible multifluid flows with large density variations. International Journal for Numerical Methods in Fluids 1998; 28:357-378. 
28. Enright D, Fedkiw R, Ferziger J, Mitchell I. A hybrid particle level set method for improved interface capturing. Journal of Computational Physics 2002; 183:83-116.

29. Hieber SE, Koumoutsakos P. A Lagrangian particle level set method. Journal of Computational Physics 2005; 210:342-367.

30. Hnat JG, Buckmaster JD. Spherical cap bubbles and skirt formation. Physics of Fluids 1976; 19:182-194.

31. Harten A, Engquist B, Osher S, Chakravarthy SR. Uniformly high order accurate essentially non-oscillatory schemes, III. Journal of Computational Physics 1987; 71:231-303.

32. Francois MM, Cummins SJ, Dendy ED, Kothe DB, Sicilian JM, Williams MW. A balanced-force algorithm for continuous and sharp interfacial surface tension models within a volume tracking framework. Journal of Computational Physics 2006; 213:141-173.

33. Lamb H. Hydrodynamics. Dover: New York, 1945.

34. Sussman M, Smereka P. Axisymmetric free boundary problems. Journal of Fluid Mechanics 1997; 341:269-294. 